\title{
Time-Dependent Models for the Afterglows of Massive Black Hole Mergers
}

\author{
Takamitsu Tanaka and Kristen Menou \\ Department of Astronomy, Columbia University, 550 West 120th Street, New York, NY 10027
}

\begin{abstract}
The Laser Interferometer Space Antenna (LISA) will detect gravitational wave signals from coalescing pairs of massive black holes in the total mass range $\left(10^{5}-\right.$ $\left.10^{7}\right) /(1+z) M_{\odot}$ out to cosmological distances. Identifying and monitoring the electromagnetic counterparts of these events would enable cosmological studies and offer new probes of gas physics around well-characterized massive black holes. Milosavljević \& Phinney (2005) proposed that a circumbinary disk around a binary of mass $\sim 10^{6} M_{\odot}$ will emit an accretion-powered X-ray afterglow approximately one decade after the gravitational wave event. We revisit this scenario by using Green's function solutions to calculate the temporal viscous evolution and the corresponding electromagnetic signature of the circumbinary disk. Our calculations suggest that an electromagnetic counterpart may become observable as a rapidly brightening source soon after the merger, i.e. several years earlier than previously thought. The afterglow can reach super-Eddington luminosities without violating the local Eddington flux limit. It is emitted in the soft X-ray by the innermost circumbinary disk, but it may be partially reprocessed at optical and infrared frequencies. We also find that the spreading disk becomes increasingly geometrically thick close to the central object as it evolves, indicating that the innermost flow could become advective and radiatively inefficient, and generate a powerful outflow. We conclude that the mergers of massive black holes detected by LISA offer unique opportunities for monitoring on humanly tractable timescales the viscous evolution of accretion flows and the emergence of outflows around massive black holes with precisely known masses, spins and orientations.
\end{abstract}

Subject headings: accretion, accretion disks — black hole physics — gravitational waves - quasars: general

\section{Introduction}

Massive black holes (MBHs) are abundant at all observable redshifts, manifesting themselves most often as active galactic nuclei (AGN) and quasars (Kormendy \& Richstone 1995; 
Richstone et al. 1998). A merger of a pair of galaxies each containing a MBH will result in the formation of a MBH binary (Begelman et al. 1980). Given the hierarchical structure formation history of the universe, and that most or all formed galaxies appear to harbor a MBH in their nuclei (e.g., Magorrian et al. 1998), there should be many MBH binaries formed throughout cosmic time (Haehnelt 1994; Menou et al. 2001; Volonteri et al. 2003; Wyithe \& Loeb 2003; Sesana et al. 2007; Tanaka \& Haiman 2009). Once compact, these binaries rapidly lose orbital energy via gravitational radiation (GW) and coalesce. The Laser Interferometer Space Antenna (LISA) will detect gravitational waves resulting from MBH mergers of binaries with total mass $M=\left(10^{5}-10^{7}\right) /(1+z) M_{\odot}$ out to redshift $z \sim 20$ (e.g., Schutz 2009). LISA is expected to be able to constrain the individual redshifted mass $(1+z) M$, the mass ratio $q \leq 1$, spins of the MBHs, and the luminosity distance of the source with precision, thereby providing an unprecedented test of general relativity and probing the assembly history and demography of MBHs (e.g., Hughes 2002; Vecchio 2004; Lang \& Hughes 2006). However, the space-based detector will be unable to measure the redshift (due to the fundamental degeneracy between source mass and redshift) or the precise angular location (due to the fact that sources are located using the modulation of the signal due to orbital motion and separation of the detector elements) of the source (Cutler 1998; Holz \& Hughes 2005; Kocsis et al. 2006, 2007; Lang \& Hughes 2008).

The coalescence of a MBH binary is not by itself expected to emit an observable electromagnetic (EM) signal. If, however, MBH mergers have associated and readily identifiable EM signatures, their detection would complement LISA by helping determine source redshifts and locations on the sky (Holz \& Hughes 2005; Kocsis et al. 2006; Bloom et al. 2009; Phinney 2009). An obvious candidate to effect such emission is the abundant gas linked to galactic MBHs. Rapid, sustained gas accretion onto MBHs is required to explain the luminosities and number of observed AGN and quasars. Numerous studies suggest that a gas-rich environment aids the formation of close $\mathrm{MBH}$ binaries in recently merged galaxies (Escala et al. 2005; Dotti et al. 2007; Callegari et al. 2009; Colpi et al. 2009), and may facilitate the further shrinking of the orbit to where GW emission can enact the merger (Ivanov et al. 1999; Armitage \& Natarajan 2002; MacFadyen \& Milosavljević 2008; Cuadra et al. 2009; Lodato et al. 2009). Various types of interactions between MBH binaries and their gas environments have been proposed as viable mechanisms for observable EM emission. Numerous studies have calculated the EM emission from the response of the gas disk to the mass loss and gravitational recoil (Peres 1962; Bekenstein 1973) effects which accompany the merger process (e.g., Bode \& Phinney 2007; Schnittman \& Krolik 2008; Lippai et al. 2008; Shields \& Bonning 2008; O’Neill et al. 2009; Megevand et al. 2009; Corrales et al. 2009; Rossi et al. 2009; Anderson et al. 2009). Chang et al. (2009) have suggested a luminous EM signal from tidal and viscous excitation of fossil gas trapped inside the binary's orbit. Krolik (2010) pointed out that even small amounts of gas that is present in the immediate vicinity of a merging binary can power short afterglows on Eddington luminosity scales. Kocsis \& Loeb (2008) 
have proposed an infrared afterglow from the dissipation of GW through a surrounding gas disk (see, however, Krolik 2010). Palenzuela et al. (2009a,b); Mösta et al. (2009) have emphasized the possibility of variable emission due to the perturbation and enhancement of the local EM fields. Haiman et al. (2009a) have raised the possibility of detecting the binary as a periodic variable source prior to the merger.

In this paper we revisit the accretion afterglow mechanism proposed by Milosavljević \& Phinney (2005; hereafter MP05; see also Liu et al. 2003; Liu 2004) for a geometrically thin circumbinary disk. We summarize their model as follows:

1. The tidal torques from the binary open a gap in the circumbinary gas. The gas inside the gap accretes, while the gas outside is held at bay by tidal torques. What results is a circumbinary disk with the binary inside the central cavity. Because it is largely "missing" the inner, highest-temperature region, the disk cannot easily produce thermal X-rays via viscous dissipation (see, however, Chang et al.2009). The system can remain in this configuration for an extended period of time (Ivanov et al. 1999; MacFadyen \& Milosavljević 2008) as the timescale for the extraction of angular momentum from the binary's orbit is long.

2. Once the binary reaches a semimajor axis $a \sim 100 G M / c^{2}$, it rapidly loses orbital angular momentum and energy via GW emission and the orbit closes faster than the surrounding gas can viscously follow (see also Armitage \& Natarajan 2005; Haiman et al. 2009a; Chang et al. 2009). The binary coalesces, producing the GW signature detectable by LISA.

3. The gas (no longer held back by binary torques) reaches the center of the cavity by viscously spreading, and the corresponding accretion flow, deep in the potential well of the $\mathrm{MBH}$ remnant, emits an X-ray afterglow.

By taking the difference between the time it takes the binary to merge and the time it takes for the bulk of the gas at the cavity edge to reach the central remnant, MP05 estimated that the X-ray afterglow would occur $t_{\mathrm{EM}} \sim 7\left(M / 10^{6} M_{\odot}\right)^{1.3}(1+z)$ yr after the GW signal (in the observer's rest frame).

In this study, we use the term "accretion afterglow" to denote the emission described above. We stress that this is a distinct mechanism from the various afterglow mechanisms that are powered by the response of the accretion disk to the mass loss and/or recoil of the central MBH remnant (references above). In the latter scenarios, mass loss and/or recoil introduce additional eccentricities in the gas orbits, and the emission is powered by the circularization of the orbits and shock/wave dissipation. In the accretion afterglow scenario considered by MP05 and in this paper, the emission results from the deepest parts of the $\mathrm{MBH}$ potential well becoming accessible to the circumbinary disk as the binary shrinks and merges. Before the merger the disk is deficient 
in the hard UV and X-ray frequency range because of the central cavity, while after the merger this high-frequency emission emerges as the cavity fills with gas. Although distinct, the various classes of emission mechanisms considered are not entirely unrelated. For example, mass loss introduces a roughly constant eccentricity everywhere in the disk, and the resulting luminosity of a circularization-powered afterglow (references above) would depend delicately on the innermost density profile of the circumbinary disk at the time of merger (see Corrales et al. 2009 for a comparison of afterglows for different density profiles). A high surface density for the innermost disk (where the available specific orbital energy is highest) at merger would enhance the mechanism emphasized by O'Neill et al. (2009) while it would suppress the pre- and post-merger emission contrast considered in this paper; a low surface density would have the reverse consequences for the two mechanisms.

The purpose of this work is to investigate the onset and time dependence of the afterglow in greater detail. We use an idealized semi-analytic framework to model the time dependence of the electromagnetic signal. We present explicit, integral solutions for the time evolution of onedimensional (viz. geometrically thin and azimuthally symmetric), viscous Keplerian disks. (See similar work by Shapiro 2010.) Our calculation method is valid insofar as the disk kinematic viscosity can be described as a simple function of radius, and as long as after decoupling the inner gas is minimally affected by the gravitational torques from the binary. This last point is worth emphasizing, as in general the decoupling of the binary from the gaseous influence does not guarantee that the gas is entirely free from the binary influence. Even after GW emission has become the dominant mechanism driving the orbital evolution of the binary, the binary's tidal torques can still influence any gas that is able to remain in the vicinity of the binary's orbit.

This paper is organized as follows. In $\$ 2$ we review the disk properties as the GW-driven closing of the binary decouples from the viscosity-driven spreading of the circumbinary disk, and introduce a semi-analytic model for the subsequent evolution of the disk's surface density. Various derivations and intermediate results used in this section are provided in the Appendices $\mathrm{A} \& \mathrm{~B}$. We present in $\$ 3$ the predictions of the model for the light curve and spectral evolution of the resulting electromagnetic emission. We also discuss in that section the possibility that the X-ray afterglow could be reprocessed by the outer disk, as well as possible effects of advection as the inner disk becomes geometrically thick. We conclude in $\$ 4$,

\section{Modeling the Binary-Disk System}

Below, we first discuss the properties introduced by MP05 for the evacuated circumbinary gas disk at the time when the evolution of the binary orbit becomes dominated by $\mathrm{GW}$ emission and thus decoupled from the evolution of the surrounding disk, which evolves viscously (see also 
Haiman et al. 2009b). We then describe the semianalytic integral formalism for the viscous evolution of an accretion disk with an arbitrary initial surface density profile. Additional derivations and intermediate results for both the disk properties and the semianalytic model are detailed in Appendices $\mathrm{A}$ and $\mathrm{B}$, respectively. Throughout this paper, $R$ is the radial distance from the center of mass of the binary in the plane of its orbit; $a$ is the binary's semimajor axis; $M$ is the total mass of the binary; and $q \leq 1$ is the binary mass ratio. The quantities $c, G, m_{p}, h, k, \sigma$ and $\sigma_{\mathrm{T}}$ are the speed of light, the gravitational constant, the mass of the proton, Planck's constant, the Boltzmann constant, the Stefan-Boltzmann constant and the Thomson cross section, respectively. Times, frequencies and rates are in the rest frame of the binary, unless noted otherwise.

\subsection{The Circumbinary Disk at Decoupling}

The "cold" gas in the nucleus is assumed to settle into a geometrically thin, rotationally supported disk. In this paper, we are concerned with the properties of the innermost disk and the binary in the very last stages of its evolution. We assume that the angular momentum of the disk is aligned with the orbital angular momentum of the binary (i.e., no warps; Ivanov et al. 1999; however we shall revisit the possibility of warping later), and that the binary has been circularized by interactions with the surrounding gas (see Armitage \& Natarajan 2005, MacFadyen \& Milosavljević 2008 and Cuadra et al. 2009 for possible complications).

The disk is able to evolve locally on length scales $\sim R$ on the viscous timescale

$$
t_{\mathrm{visc}}(R)=\frac{2}{3} \frac{R^{2}}{\nu}
$$

where $\nu(R)=\frac{2}{3} \alpha P_{\text {gas }} /(\rho \Omega)=\frac{2}{3} \alpha k T /\left(\mu m_{\mathrm{p}} \Omega\right)$ and $P_{\text {gas }}, \rho, T$ and $\mu=0.6$ are the pressure, density, temperature and mean molecular weight of the gas, respectively. The quantity $\Omega$ is the angular velocity, assumed to be approximately Keplerian: $\Omega \approx \sqrt{G M / R^{3}}$. The parameter $\alpha$ is the viscosity parameter in the disk model of (Shakura \& Sunyaev 1973). We follow MP05 in adopting a viscosity prescription where $\nu$ scales only with the gas pressure, as opposed to the total (gas, radiation plus magnetic) pressure. This choice is motivated by calculations that suggest that disks where viscosity scales with the total pressure may be thermally unstable (Shakura \& Sunyaev 1976; Pringle 1976; see, however, Hirose et al. 2009b).

At a distance of $R \sim 2 a$, a gap is opened in the circumbinary disk through tidal interactions (see, e.g., Artymowicz et al. 1991, MacFadyen \& Milosavljević 2008 and Haiman et al. 2009b). In

\footnotetext{
${ }^{1}$ However, radiation pressure dominated disks may still be viscously unstable (Lightman \& Eardley 1974; Piran 1978; Hirose et al. 2009a). Because the afterglow mechanism discussed in this paper takes place in the radiationdominated part of the disk, the findings reported here are contingent on the answer to this open theoretical question.
} 
this region the input of angular momentum from the tidal torques exerted by the binary counteracts the viscous spreading of the disk. For simplicity, we neglect the small amount of fossil gas that may be present inside the binary's orbit (Chang et al. 2009). The gap, at first annular, becomes a roughly circular central cavity after the inner gas is consumed. The boundary between the cavity and the disk is characterized by a steep surface density gradient, a pile-up of gas caused by tidal decretion in the vicinity of the binary's orbit and viscous accretion outside the decreting region. Whereas a steady-state accretion disk has a mass accretion rate $\dot{M}(R)=3 \pi \nu \Sigma$ that is constant with radius, a decretion disk with a steady inner torque instead satisfies constant viscous torque, i.e. $3 \pi R^{2} \nu \Sigma \Omega=$ constant (e.g., Rudak \& Paczynski 1981; Pringle 1991). It is a priori unclear how close to accretion or decretion a certain system is at decoupling, as this will depend on the system parameters and its past accretion history (e.g., Ivanov et al. 1999; Chang et al. 2009). We thus consider various possibilities for the circumbinary disk density profile at decoupling. Some of the innermost gas may be still able to accrete across the binary's orbit in narrow streams, but the accretion rate is expected to be only a small fraction of that of a comparable accretion disk around a single black hole (e.g., Lubow et al. 1999; Günther et al. 2004; Hayasaki et al. 2007, 2008; MacFadyen \& Milosavljević 2008).

The timescale for the binary separation to shrink via GW emission is

$$
t_{\mathrm{GW}}(a) \equiv \frac{a}{d a / d t}=\frac{5}{16} \frac{c^{5}}{G^{3} M^{3}} \frac{a^{4}}{\zeta} \approx 4.90 \mathrm{yr} \times\left(\frac{a}{100 G M / c^{2}}\right)^{4} \zeta^{-1}
$$

where $\zeta \equiv 4 q /(1+q)^{2}$ is the symmetric mass ratio scaled to unity for equal-mass binaries. Equation (2.2) is valid for binaries with non-extreme mass ratios and circular orbits. Because $t_{\mathrm{GW}} \gg t_{\mathrm{visc}}$ initially in the circumbinary disk, the gas is able to respond promptly to the gradual and relatively slow shrinking of the binary, probably maintaining a roughly constant geometric ratio between the disk inner edge and the orbital semimajor axis, i.e. $\lambda \equiv R /(2 a) \sim 1$.

Because the disk viscosity $\nu$ is a weak function of radius outside the inner edge (see Appendix A), roughly speaking the viscous time there scales as $t_{\mathrm{visc}}(R) \propto R^{2}$. As the binary closes, $t_{\mathrm{GW}}(a) \propto a^{4}$ will inevitably become shorter than the disk viscous time at the inner edge, and the binary will begin to close faster than the bulk of the gas at the inner edge can follow. The subsequent evolution of the binary is driven by GW emission and thus causally decoupled from that of the disk. In the absence of binary torques, the gas at the cavity edge would fill the cavity in a time $\sim \beta t_{\text {visc }}$, where the approximate boost factor $\beta \sim 0.1$ was introduced by MP05 to account for the limit of a very steep surface density gradient at the edge (Lynden-Bell \& Pringle 1974). Decoupling occurs, then, when $t_{\mathrm{GW}}(a) \sim \beta t_{\mathrm{visc}}(2 \lambda a)$. This condition, along with the assumption that the viscously dissipated energy is locally emitted as thermal radiation, specifies the various properties of the inner edge of the disk described by MP05. We closely reproduce their results in Table 1, and detail the various intermediate calculations in Appendix A. Note that while we recover the parameter dependencies 
exactly, the magnitudes of our disk properties differ somewhat from those of MP05. This is due to differences in how we have calculated the thermal structure of the disk, the most significant being that the flux expression in MP05 is a factor of two lower than ours (compare their equation [2] and preceding formula for $F_{\nu}$ with our equation [(3.4)] below). Throughout the rest of this paper, we use the subscript " 0 " to denote the value of a quantity at decoupling. When applied to a disk quantity with radial dependence, the subscript shall also denote the value at the inner edge of the disk. For example, $a_{0}$ is the semimajor axis of the binary orbit at decoupling and $\Omega_{0}$ is the angular velocity at the inner edge of the disk at decoupling.

The surface density at the inner edge of the circumbinary disk at decoupling cannot be determined through scaling arguments alone. However, we expect it to be greater than the value expected for a steady thin accretion disk. Just outside the edge, the gas may resemble a decretion disk, with $3 \pi \nu \Sigma \propto R^{-1 / 2}$ approximately, because of mass accumulation. Sufficiently far from the binary and its tidal torques, we expect the gas to behave more like a steady thin accretion disk, with a local mass supply rate $\dot{M}=3 \pi \nu \Sigma$ roughly constant and determined by external conditions (e.g., Ivanov et al. 1999; Chang et al. 2009). It follows that if $3 \pi \nu \Sigma$ is monotonic outside the edge then it must be greater than the outer mass supply rate of the disk. Therefore, we parametrize the surface density at the inner edge via the arbitrary relation $\Sigma_{0}=S \dot{M}_{\mathrm{Edd}} /\left(3 \pi \nu_{0}\right)$, where $S$ is a dimensionless parameter and $\dot{M}_{\mathrm{Edd}}=4 \pi G M m_{\mathrm{p}} /\left(\eta c \sigma_{\mathrm{T}}\right)$ is the Eddington accretion rate onto a $\mathrm{MBH}$ of mass $M$ for a radiative efficiency $\eta=0.1$. Effectively, $S$ is the product of the gas supply rate onto the disk in Eddington units and the enhancement of the local surface density at the disk edge due to the binary torques and mass accumulation. 2 In general, $S$ can exceed unity even if the disk mass supply rate and the local accretion rate are both sub-Eddington.

\subsection{A Simple Model for the Viscous Evolution}

Following decoupling, the binary outruns the surrounding gas to the center and merges in a time $t_{\mathrm{GW}, 0} / 4$. MP05 estimated that an accretion-powered X-ray afterglow would take place when the central cavity is filled, viz. after a time $t_{\mathrm{EM}}=\beta t_{\mathrm{visc}, 0}-t_{\mathrm{GW}, 0} / 4 \sim(3 / 4) t_{\mathrm{GW}, 0}$ after the $\mathrm{GW}$ event. Below, we revisit this estimate by calculating the time dependence of the signal with a simple model for the disk evolution. We proceed by assuming that following decoupling the binary's orbit closes faster than the gas can follow, viz. viscosity is the only source of torque in the disk and

\footnotetext{
${ }^{2}$ For practical purposes $S$ is equivalent to the parameter " $\ddot{m}$ " used in MP05. We avoid using the latter notation to prevent confusion with the actual local accretion rate, which is discussed below in $\$ 2.2$ Also note that $\Sigma_{0}$ and $S$ do not relate linearly (see Table 1). The relationship is more complex because the disk gas viscosity $\nu_{0}$ and the decoupling orbital radius $a_{0}$ also depend on $S$ through the midplane temperature.
} 
the gas orbits remain circular and Keplerian. Numerical simulations (e.g., Armitage \& Natarajan 2005; Hayasaki et al. 2007; MacFadyen \& Milosavljević 2008; Cuadra et al. 2009) of MBH circumbinary disks indicate that both the eccentricity and the deviation of the angular velocity from the Keplerian value are small (of order a few percent) at the radius where the binary tides truncate the disk. Likewise, the mass reduction of the binary at merger due to GW emission will only introduce orbital eccentricities (roughly equal to the fractional mass loss) of $\gtrsim 10^{-2}$ (Tichy \& Marronetti 2008; O'Neill et al. 2009; Reisswig et al. 2009). At radii and binary masses of interest here, orbital speeds are too great for the gas to be significantly affected by gravitational recoil of the MBH remnant: $\Omega \sim \sqrt{R c^{2} / G M} c \gg v_{\text {recoil }} \sim 300 \mathrm{~km} \mathrm{~s}^{-1}$. After the binary has merged, the central potential is that of a rotating black hole and circular orbits near the center will have somewhat super-Keplerian angular velocities. Approximate "pseudo-Newtonian" expressions of the Kerr potential (e.g., Artemova et al. 1996) suggest that for a merger remnant with less than maximal spin, the deviation from the Keplerian value is of order ten percent at the radius of innermost stable circular orbit and falls off as roughly $\propto R^{-1}$. All of these modest deviations and perturbations are neglected in our models.

The viscous evolution of a geometrically thin, cylindrically symmetric Keplerian disk can be described by the standard equation (e.g. see Pringle 1981 and Frank et al. 2002)

$$
2 \pi R \frac{\partial}{\partial t} \Sigma(R, t)=\frac{\partial}{\partial R}\left[2 R^{1 / 2} \frac{\partial}{\partial R}\left(3 \pi \nu \Sigma R^{1 / 2}\right)\right]
$$

where $\Sigma$ is the surface density of the disk and the innermost derivative on the right-hand side describes the angular momentum gradient. The left-hand side of (2.3) describes the rate of change of the radial mass distribution, while the right-hand side gives the radial gradient of the local mass flow

$$
\dot{M}=3 \pi \nu \Sigma(1+2 m)
$$

where $m(r) \equiv \partial \ln (\nu \Sigma) / \partial \ln R$. In the above equation, the dimensionless factor in parentheses equals unity for steady-state accretion solutions $(m \approx 0)$ while it vanishes in steadily decreting solutions $(m \approx-1 / 2)$.

If the kinematic viscosity is a function of radius only, equation (2.3) is a linear diffusion equation. In the special case $\nu \propto R^{n}$, the solution for subsequent viscous evolution (see, e.g. Lynden-Bell \& Pringle 1974; a derivation is provided in our Appendix (B) is given by

$$
\Sigma(r, t)=\int_{0}^{\infty} G\left(r, r^{\prime}, t\right) \Sigma\left(r^{\prime}, t=0\right) d r^{\prime},
$$


where

$$
\begin{aligned}
G\left(r, r^{\prime}, t\right)=\frac{2-n}{\tau} & r^{-1 / 4-n} r^{5 / 4} I_{1 /(4-2 n)}\left(\frac{2 r^{1-n / 2} r^{1-n / 2}}{\tau}\right) \\
& \times \exp \left(-\frac{r^{\prime 2-n}+r^{2-n}}{\tau}\right)\left\{1-\sqrt{\frac{r_{*}}{r}} \exp \left[-\frac{\left(r-r_{*}\right)^{2-n}}{\mathcal{R}\left(\tau-\tau_{\text {merge }}\right)}\right]\right\}
\end{aligned}
$$

is the Green function, $\Sigma(r, t=0)$ is the initial surface density profile (at decoupling), $I_{m}$ is the modified Bessel function of the first kind, $\mathcal{R}$ is the ramp function, and $r \equiv R / R_{0}, r^{\prime} \equiv R^{\prime} / R_{0}$ and $\tau \equiv 8(1-n / 2)^{2} t / t_{\mathrm{visc}, 0}$ are dimensionless variables. At decoupling, $\tau=0$; the binary merges when $\tau=\tau_{\text {merge }}=2 \beta(1-n / 2)^{2}$; roughly speaking, gas concentrated at $r=1$ diffuses to the center in a time $\tau \sim 1$ (hence the value $\beta \sim 0.1$ adopted by MP05).

The second term in the curled brackets of equation (2.6) accounts for the fact that, after merger, the $\mathrm{MBH}$ remnant imposes a zero-torque boundary condition at some finite radius $R_{*}=$ $R_{0} r_{*}$, which we associate with the remnant's innermost radius for marginally stable circular orbits 3 $\left(R_{\mathrm{ISCO}}\right)$. This radius depends on the MBH spin as $a_{\text {spin }}=(1 / 3) \sqrt{R_{\mathrm{ISCO}} c^{2} / G M}\left(4-\sqrt{3 R_{\mathrm{ISCO}} c^{2} / G M-2}\right)$ (Bardeen 1970), where $-1 \leq a_{\text {spin }} \leq 1$ is the standard dimensionless spin parameter of the MBH. For test particle orbits, one finds $R_{*}=6 G M / c^{2}$ if $a_{\text {spin }}=0$, and $R_{*}=G M / c^{2}$ if $a_{\text {spin }}=1$ and the spin of the hole is perfectly aligned with the test particle's orbital angular momentum. Immediately after the merger, the $\mathrm{MBH}$ remnant is expected to have moderately high but non-maximal spin for a wide range of physically plausible scenarios (e.g. Hughes \& Blandford 2003; Baker et al. 2004; Berti \& Volonteri 2008; see however the "chaotic" accretion scenario of King \& Pringle 2006, which predicts lower MBH spins). We thus adopt the intermediate value $R_{*}=3 G M / \mathrm{c}^{2}$, which is accurate to within $\sim 20 \%$ in the range of spin $0.65 \lesssim a_{\text {spin }} \lesssim 0.9$. Our solutions given by equation (2.6) depend only on the initial surface density profile (which may be arbitrarily complex and need not be differentiable), as well as on the values of $n \leq 2$ and $r_{*}$.

Figure 1 shows time-dependent solutions calculated following equation (2.5) for two different initial surface density profiles at decoupling (upper left panel). In solid lines, we evolve for demonstrative purposes the plainest possible model: a surface density profile obeying the decretion power-law $\Sigma \propto r^{-1 / 2-n}$, truncated by a step function at the cavity edge. We also evolve a second, more plausible profile (dotted lines):

$$
\frac{\Sigma(r, 0)}{\Sigma_{0}}=\frac{1}{r^{n+1 / 2}+\exp [-11.9 \lambda(r-1)]} .
$$

This analytic profile is inspired by the hydrodynamic simulation results of MacFadyen \& Milosavljević (2008), who found an azimuthally averaged inner surface density profile with $\Sigma(R \lesssim 2 a) \propto \exp (-5.95 R / a)$

\footnotetext{
${ }^{3}$ See, however, Krolik \& Hawley (2002), who showed that there are several ways to define an effective inner radius, some of which are quite different from $R_{\mathrm{ISCO}}$.
} 
for a thin disk around an equal-mass binary. Equation (2.7) reproduces this exponential behavior for $r \lesssim 1$ and the decretion power-law behavior $\Sigma \approx \Sigma_{0} r^{-1 / 2-n}$ for $r \gtrsim 1$. Note that this profile does not peak at the inner edge $r=1$, nor is its peak value equal to the parametrized quantity $\Sigma_{0}$. In the context of equation (2.7), the value of $\Sigma_{0}$ sets the scale for the power-law region of the surface density profile just outside the inner edge.

In Figure 1 , the physical length scale and the time with respect to the binary merger is calculated using the relations in Table 1 and the fiducial parameter values $M_{6}=q=\alpha_{-1}=\beta_{-1} \lambda=s=\theta_{0.2}=$ 1. We take as $n=0.4$ as our fiducial viscosity power-law. For our disk solutions, this value leads to agreement between the late-time disk profile $\nu \Sigma \propto 1-\sqrt{R_{*} / R}$ and the physical viscosity prescription $\nu \propto P_{\text {gas }} /(\rho \Omega)$ of the $\alpha_{\text {gas }}$ disk. (We refer the reader to Appendix $\AA$ for a brief discussion on the parameter $n$.) In Figure 2, we also evolve a second case with $n=11 / 170 \approx 0.065$, which is consistent with $\nu \propto P_{\text {gas }} /(\rho \Omega)$ at early times just outside the inner edge for a disk that has the decretion profile $m \approx-1 / 2$ there. In both figures, we graph the profiles at decoupling (3.1 years before the merger), 1 year before merger, at merger, and 9.2 years after merger. The last snapshot corresponds to a time $\beta t_{\text {visc }, 0}$ after decoupling, our re-evaluation of MP05's nominal estimate for the onset of the X-ray afterglow.

These two figures suggest that the qualitative evolution of the disk does not depend very sensitively on $n$. The viscous evolution timescale governing the Green's function scales as (1$n / 2)^{2}$, and thus the temporal dependence on $n$ is small as long as the quantity $1-n / 2$ is of order unity. In both figures, the qualitative evolution is such that at early times and large radii $(t<$ $\left.t_{\text {visc }}(R)\right)$, the disk maintains the initial mass distribution. At late times and small radii $\left(t \gtrsim t_{\text {visc }}(R)\right)$, the disk approaches an analytic quasi-steady density profile. Before the merger, we do not specify an inner boundary condition and so the quasi-steady surface density profile satisfies $\Sigma \propto \nu^{-1} \propto R^{-n}$. After the merger, however, we assume that the MBH remnant imposes a zero-torque boundary condition at finite radius $R_{*}$, which leads to a quasi-steady surface density profile satisfying $\Sigma \propto$ $\left(1-\sqrt{R_{*} / R}\right) R^{-n}$. The two initial profiles evolve to become largely indistinguishable even before the binary merges. For a variety of initial profiles, we have confirmed that the solutions converge on timescales shorter than the time to merger if the profiles share the same value of $\lambda$, which is closely related to the location of the maximum of the surface density gradient, and have identical profiles just outside the inner edge.

We note that the physical circumbinary disk is not likely maintain a $m \approx-1 / 2$ decretion profile at radii far outside of the inner edge. Where the gas is causally detached from the binary torques and the mass accumulation of the inner edge, it is expected to have the $m \approx 0$ profile of a steady accretion disk. The simulations of MacFadyen \& Milosavljevic (2008) suggest that this transition to a steady accreting solution does not occur until the radius is at least several times the size of the central cavity. Because the viscous time scales as $t_{\text {visc }}(R) \propto R^{2-n}$, we do not expect the gas profile 
at decoupling at these outer radii to contribute to the EM afterglow on timescales of observational interest.

Despite decoupling, some gas is able to follow the binary closely. This raises the possibility that some gas could continue to partially influence the subsequent orbital evolution of the binary. Such a dynamical effect will be proportional to the gaseous mass present in the vicinity of the binary. Since the surface density profile at decoupling cannot viscously evolve significantly at radii beyond several times $R_{0}$, we choose to conservatively evaluate the maximum dynamical influence of the circumbinary gas based on the mass inside $10 R_{0}$. All of the disk profiles in Figures 1 and 2 have a total mass inside $R<10 R_{0}$ of

$$
M_{\text {disk }}\left(R<10 R_{0}\right) \sim 10^{-5} M \times \alpha_{-1}^{-1.36} S^{0.02} \lambda^{2.8} M_{6}^{1.32}(\beta \zeta)^{0.7} \theta^{-0.34},
$$

where the parameter dependencies above are calculated from Table 1 and Appendix $\mathrm{A}$ using the fact that $M_{\text {disk }} \propto \Sigma_{0} \lambda^{2} a_{0}^{2}$. The low, conservative value of the disk to binary mass ratio in equation (2.8) suggests that the disk is unlikely to have a dynamical effect on the binary merger, and to contaminate the GW signal at levels detectable by LISA. (Note, however, the scenario of Armitage \& Natarajan 2005, in which the disk can imprint LISA-observable perturbations on the binary's orbit prior to the decoupling time.) However, such gas may still contribute an observable EM signature because the available specific gravitational energy is large (Chang et al.2009; Krolik 2010).

Even if the gas cannot influence the binary, it is possible for the binary to continue to influence the evolution of the innermost gaseous disk, even after decoupling. Our models assume that the evolution of the gas and that of the binary can effectively be treated independently, i.e. that the region where the potential is non-Keplerian shrinks faster than the gas can follow. Although this is a useful first approximation, it is not strictly guaranteed by a decoupling criterion based on GW and viscous timescales, as defined earlier. Indeed, our solutions allow for gas as dense as $\gtrsim 10^{-2} \Sigma_{0}$ to flow to $R \lesssim a(t)$ prior to the merger (see, e.g., Figure $1 \mathrm{~b}$ ). To understand why this happens, one can compare the radial gas velocity $V_{R}=-\dot{M} /(2 \pi R \Sigma)=3(1+2 m) \nu /(2 R)$ with the shrinking rate of the hypothetical disk edge, $d(2 \lambda a) / d t$. The decoupling condition $a_{0} /(d a / d t)_{0}=\beta t_{\text {visc }, 0}$ directly implies $d(2 \lambda a) / d t=\beta^{-1} R_{0} / t_{\mathrm{visc}, 0}=3 \beta^{-1} \nu_{0} /\left(2 R_{0}\right)$. We thus find that the nominal radial velocity (ignoring binary torques) at the inner edge at decoupling is given by the following expression:

$$
V_{R, 0}=0.1 \beta_{-1}\left(1+2 m_{0}\right) \frac{d(2 \lambda a)}{d t} \text {. }
$$

Above, $m_{0}$ is the index $\partial \ln (\nu \Sigma) / \partial \ln R$ evaluated at the disk edge, which in principle may be much greater than unity. For sufficiently steep surface density profiles, the innermost gas can continue to flow into the binary's vicinity 4 even after decoupling as defined earlier. Thus, the binary

\footnotetext{
${ }^{4}$ In fact, because our viscosity prescription is not causal, $V_{R}$ can be supersonic for large positive values of $m_{0}$. Flow
} 
can continue to open a gap in the disk for some time until the binary is truly able to outrun the surrounding gas. This happens when the binary's orbital radius has shrunk below the decoupling value $a_{0}$ by a factor $\left[0.1 \beta_{-1}(1+2 m)\right]^{1 /(n+2)}$. The profiles given by the analytic form in equation (2.7) have $m_{0} \gtrsim 10$ for each of the viscosity prescriptions shown in Figures 1 and 2. We thus estimate the binary can outrun the gas in these profiles when $a \lesssim 0.8 a_{0} \beta_{-1}^{-1 /(n+2)}$, at a time approximately $1 M_{6}^{1.3} \mathrm{yr}$ before the merger. This suggests that the time when the binary torques cease to influence the circumbinary gas may be as late as $\sim 0.05 t_{\mathrm{visc}, 0}$ before the merger.

To evaluate the possible effect of the binary's continued influence on the gas after formal decoupling, we consider a scenario where the inner gas has maintained an exponential inner density profile similar to the one in equation (2.7), scaled to the new semimajor axis of the binary, until one year before the merger as estimated above. This revised configuration is shown as dashed lines in Figures 1 and 2, It is evolved viscously in the same way as the other two scenarios in panels (c) and (d) of each figure. While the first two classes of profiles represent the assumption that the binary ceased to tidally interact with the circumbinary gas at decoupling $\left(t_{\mathrm{GW}}=\beta t_{\mathrm{visc}}\right)$, the third class of profiles is evolved assuming the tidal interaction continues for a while longer after decoupling, until the binary is able to truly outrun the disk edge as estimated above. We find that the three types of profiles are unlikely to be observationally distinguishable from each other (compare longdashed and dotted lines in Panels b, c, d in Figures 1 1and 2); there are small differences until merger time, but they are quickly wiped clean by the boundary condition associated with the single $\mathrm{MBH}$ remnant. Figures 1 and 2 illustrate that neither the density gradient at the inner edge at decoupling nor the subsequent gas-binary interaction inside this radius are likely to have an appreciable effect on the disk evolution on timescales of interest. At the level of accuracy of our idealized models, we do not expect either of these factors to affect too strongly the observable properties of the viscously spreading disk, to which problem we now turn.

\section{Observable Features of the Time-Dependent Afterglow}

\subsection{Bolometric Light Curve}

The power per unit area viscously dissipated from each face of a thin Keplerian disk is equal to $F \sim(9 / 8) \nu \Sigma \Omega^{2}$. Substituting our expressions for $\nu \propto r^{n}$ and $\Omega \propto r^{-3 / 2}$, and ignoring advective

speeds are, however, generally subsonic for realistic $\Sigma$ profiles, as long as $\partial \ln \Sigma / \partial \ln R \lesssim 10^{3}$ at decoupling. Note that the step-function profile shown in Figure 1 a) rapidly evolves to a profile with subsonic radial speeds throughout. 
loss, the power radiated by the disk is

$$
\begin{aligned}
L_{\mathrm{visc}}(t) & \approx \frac{9}{4} \nu_{0} \Omega_{0}^{2} \int \Sigma(r, t) 2 \pi r^{n-2} d r \\
& \approx 0.059 L_{\mathrm{Edd}} \times \alpha_{-1}^{0.34} S^{1.2} \lambda^{-1.7} M_{6}^{-0.08}\left(\beta_{-1} \zeta\right)^{-0.42} \theta^{0.08} \int \frac{\Sigma(r, t)}{\Sigma_{0}} r^{n-2} d r,
\end{aligned}
$$

where $L_{\mathrm{Edd}} \equiv 4 \pi c G m_{\mathrm{p}} M / \sigma_{\mathrm{T}}$ is the Eddington luminosity for an object with a mass equal to that of the binary. The dissipated power depends weakly on $\alpha$ and $\theta$, scales roughly linearly with the binary mass, and is most sensitive to the gas distribution parameters $S$ and $\lambda$. At decoupling, the second integral in equation (3.1) evaluates to a value $\approx 1 /(1-m)$, and thus the initial luminosity of the disk at decoupling is expected to be $\sim 0.04 L_{\text {Edd }}$ for fiducial parameters and $m \approx-1 / 2$. In integrating equation (3.1) before the binary merger, we do not calculate emission from radii inside $R<2 \lambda a(t)$. In this region, the potential is highly time dependent and non-axisymmetric, and the assumption that the gas is in nearly circular Keplerian orbits about the binary's center of mass breaks down. Hydrodynamical simulations (e.g., MacFadyen \& Milosavljević 2008; Hayasaki et al. 2008; Cuadra et al. 2009) suggest that the gas at these radii will form relatively dim, quasi-periodic accretion flows around the individual MBHs. We do not address these circumsecondary and circumprimary accretion flows in this paper. The potential role of circumprimary gas inside of the binary's orbit is discussed by Chang et al. (2009).

In Figure 3 we present several bolometric rest-frame light curves for the evolving disk, for three different values of the viscosity power-law index $n$, using equation (2.7) for the surface density profile at decoupling. We show light curves for two different surface density profiles immediately outside the disk edge at decoupling: a decretion-like disk ( $m=-1 / 2$; thick lines), and an accretion-like disk ( $m=0$; thin lines). The vertical scale is the brightening $L_{\mathrm{visc}}(t)$, in units of the disk luminosity at decoupling $L_{0}$, while the horizontal scale is the time relative to the merger. In each case, the dissipated luminosity increases steadily by over an order of magnitude within $\sim 20 \mathrm{yr}$ of the merger. There is significant evolution before and after our re-evaluation of MP05's estimate for the onset for the afterglow, $t_{\mathrm{EM}}=(3 / 4) t_{\mathrm{GW}, 0} \sim 9 M_{6}^{1.3} \mathrm{yr}$ after the merger.

The sudden brightening that accompanies the merger in Figure 3 is due to the fact that we only integrate the circumbinary disk emission outward of the radius $R=2 \lambda a(t)$, as described above: since the binary will merge within $\sim 48$ hours once it reaches a separation of $\sim 50 G M / c^{2}$, the integrated area rises sharply just before the merger. This is a somewhat artificial ingredient of our model. We note, however, that at merger the depth of the potential well near the center of mass does increase drastically, as does the area where the gas is free to spread viscously. This sudden change in the central potential well could be associated with significant emission, for instance if even trace amounts of gas remain present between the components of the merging binary (Chang et al. 2009).

The disk emission can exceed $L_{\text {Edd }}(M)$, the Eddington luminosity limit associated with the 
binary mass $M$, without violating local Eddington flux limits. This result may appear contradictory, but it can be understood as follows. The Eddington flux limit associated with the vertical gravitational field of the disk (ignoring self-gravity) is

$$
F_{\mathrm{Edd}, z}=\left[1+\left(\frac{H}{R}\right)\right]^{-1 / 2} \frac{c}{\kappa_{\mathrm{es}}} \frac{G M}{R^{2}},
$$

where $H$ is the scale height of the disk and $\kappa_{\mathrm{es}} \approx 0.40 \mathrm{~cm}^{2} \mathrm{~g}^{-1}$ is the electron-scattering opacity. The maximum luminosity of a steady disk is then $L_{z} \sim 4 \pi \int F_{\mathrm{Edd}, z} R d R$, where we have introduced an additional factor of two to account for the fact that the disk emits from two faces. The leading geometric factor in equation (3.2) is a monotonically increasing function of $H / R$. It approaches the value $H / R$ in the limit $H / R \ll 1$, is of order $\sim 0.7$ when $H / R \sim 1$, and has an asymptotic maximum of unity in the limit $H / R \rightarrow \infty$. The luminosity $L$ emitted by an annulus with inner and outer radii $R_{\text {in }}$ and $R_{\text {out }}$ is capped by the inequality

$$
L<\int_{R_{\mathrm{in}}}^{R_{\mathrm{out}}} 4 \pi \frac{c G M}{\kappa_{\mathrm{es}}} R^{-1} d R=\ln \left(\frac{R_{\mathrm{out}}}{R_{\mathrm{in}}}\right) L_{\mathrm{Edd}} .
$$

Thus, the bolometric luminosity can reach several Eddington luminosities for reasonable disk sizes.

We find that the evolution of the light curve is largely determined by the viscosity profile in the disk and has a relatively weak dependence on the surface density profile beyond the disk edge. Figure 3 suggests that the accretion luminosity of the viscously spreading inner disk may be a viable candidate for observational follow-up almost immediately after the main GW event. If the electromagnetic accretion signature associated with the spreading inner disk can be detected in the "rise" phase, the light curve may provide clues about the nature of viscosity in the accretion disk. We find that our disk solutions can develop bolometric luminosities in excess of the Eddington luminosity of the merged MBH remnant. These luminosities can be generated even if the locally dissipated flux is sub-Eddington ( $\$ 3.4$ below), and are enhanced strongly if the value of the massaccumulation parameter $S$ greater than unity.

\subsection{Spectral Evolution}

We now turn to the spectral evolution of the viscously spreading disk. Below, we present an abbreviated derivation of the thermal structure of the inner circumbinary disk and discuss its spectral features. We refer the reader to MP05 and our Appendix A for more detailed derivations.

The spectrum differs from that of an exact blackbody because the photons of different frequencies are thermalized at different depths above the disk. This thermalizing region is referred to

the "thermalization photosphere" or the "effective photosphere." The temperature at the bottom of 
the photosphere is equal to the effective temperature as seen by an observer far above the photosphere, viz. the effective optical depth above this height is of order unity. We call this temperature the photospheric temperature, $T_{\mathrm{p}}$.

There are two sources of opacity in the photosphere: electron scattering, which has the frequency-independent opacity $\kappa_{\mathrm{es}}$; and absorption, which is dominated by the bound-free process. We follow MP05 and prescribe a Kramer's functional form for the absorption opacity $\kappa_{\text {abs }, \nu} \propto$ $\rho T^{-7 / 2} f_{\nu}(\xi)$, where $f_{\nu}(\xi) \equiv \xi^{-3}\left(1-e^{-\xi}\right)$ and $\xi \equiv h \nu / k T$. We scale $\kappa_{\text {abs }, \nu}$ so that its Rosseland mean recovers the standard Kramer's bound-free opacity for solar metallicity, $1.6 \times 10^{24}\left(\rho \mathrm{cm}^{3} \mathrm{~g}^{-1}\right)(T / \mathrm{K})^{-7 / 2} \mathrm{~cm}^{2} \mathrm{~g}^{-1}$. For a wide range of system parameters, we find that scattering is the dominant source of opacity in the photosphere at the inner edge at decoupling. In this limit, $\kappa_{\mathrm{abs}, \nu} \ll \kappa_{\mathrm{es}}$, we find $\kappa_{\mathrm{abs}, \nu} \approx$ $\kappa_{\mathrm{abs}, *} f_{\nu}^{1 / 2}$, with $\kappa_{\mathrm{abs}, *} \approx 4.7 \times 10^{20} \mathrm{~cm}^{2} \mathrm{~g}^{-1} \times(\Omega \mathrm{s})\left(T_{\mathrm{p}} / \mathrm{K}\right)^{-15 / 4}$ a frequency-independent quantity. (See Appendix $\mathrm{A}$ for the intermediate calculations for the absorption opacity, including the general case allowing for $\kappa_{\mathrm{abs}, \nu} \sim \kappa_{\mathrm{es}}$.)

The so-called "graybody" flux emitted by each face of the disk is given by the expression (e.g., Rybicki \& Lightman 1986; Blaes 2004)

$$
F_{\nu} \sim \pi \frac{2 \epsilon_{\nu}^{1 / 2}}{1+\epsilon_{\nu}^{1 / 2}} B_{\nu}
$$

where $B_{\nu}\left(T_{\nu}\right)$ is the Planck function and $\epsilon_{\nu} \equiv \kappa_{\mathrm{abs}, \nu} /\left(\kappa_{\mathrm{abs}, \nu}+\kappa_{\mathrm{es}}\right) \leq 1$ is the ratio of the absorption to the total opacity. Both quantities are evaluated at $T_{\mathrm{p}}$, the temperature at the bottom of the photosphere. Since $\kappa_{\mathrm{abs}, \nu}$ is a monotonically decreasing function of $\nu$, the photosphere emits increasingly less efficiently at higher frequencies, relative to a blackbody with the same temperature. In other words, the photosphere has a higher temperature relative to a blackbody with the same radiant flux, and thus emits at higher frequencies. The expression given in (3.4) recovers the blackbody flux $F_{\nu}=\pi B_{\nu}$ in the limit where absorption is the dominant source of opacity, i.e. when $\epsilon_{\nu} \rightarrow 1$. This limit is relevant for our disk solutions, in which $\epsilon_{\nu}$ can span the full range between 0 and 1 in radii and frequencies of interest. Note that the flux expression used by MP05 is lower than ours by a factor of two.

If again advective losses are ignored, integrating the flux of equation (3.4) over frequency must give the flux viscously dissipated by half of the disk, $(9 / 8) \nu \Sigma \Omega^{2}$. We obtain

$$
\frac{F_{\text {visc }}}{2}=\int_{0}^{\infty} F_{\nu} d \nu=\Xi\left(T_{\mathrm{p}}, \Omega\right) \sigma T_{\mathrm{p}}^{4}=\frac{9}{8} \nu \Sigma \Omega^{2}
$$

where

$$
\Xi\left(T_{\mathrm{p}}, \Omega\right) \equiv \frac{15}{\pi^{4}} \int_{0}^{\infty} \frac{2 \epsilon_{\nu}^{1 / 2}}{1+\epsilon_{\nu}^{1 / 2}(\xi)} \frac{e^{-\xi} d \xi}{f_{\nu}(\xi)} \approx \frac{4}{5} \epsilon_{*},
$$


is the deviation of the bolometric flux from blackbody and $\epsilon_{*}\left(T_{\mathrm{p}}, \Omega\right) \equiv \kappa_{\mathrm{abs}, *} /\left(\kappa_{\mathrm{abs}, *}+\kappa_{\mathrm{es}}\right)<1$. The above approximation for $\Xi$ is accurate to within $10 \%$ in the range $0 \leq \epsilon_{*} \lesssim 0.9$. (See Appendix $\mathrm{A}$ for a more accurate fit to the integral.)

To calculate the spectrum, we solve equation (3.5) numerically for $T_{\mathrm{p}}$, substitute the result into equation (3.4) and integrate:

$$
L_{\nu}(R, t) \approx 2 \pi^{2} R_{0}^{2} \int \frac{2 \epsilon_{\nu}^{1 / 2}\left(\Omega, T_{\mathrm{p}}\right)}{1+\epsilon_{\nu}^{1 / 2}\left(\Omega, T_{\mathrm{p}}\right)} B_{\nu}\left(T_{\mathrm{p}}\right) r d r .
$$

The temperatures at the midplane and the bottom of the photosphere are related through

$$
T^{4}=\frac{3 \tau}{4} T_{\mathrm{p}}^{4}
$$

if the region between the two heights is optically thick and can be treated as a one-zone gray atmosphere. Here $\tau=\theta \kappa_{\text {es }} \Sigma$ is the scattering-dominated optical thickness between the two heights, and $\theta \leq 1$ is a porosity correction factor. We adopt MP05's interpretation of the simulation results of Turner (2004) and use $\theta=0.2$ as our fiducial value, and define $\theta_{0.2} \equiv \theta / 0.2$.

Neglecting the advected flux, and in the limit where the circumbinary disk is dominated by radiation pressure and electron scattering, we may estimate the frequency for the peak monochromatic flux at decoupling, and at late times after the merger. At decoupling, most of the emission comes from the inner edge, and the spectrum in equation (3.7) peaks at the Wien frequency $\xi=h \nu / k T_{\mathrm{p}, 0} \approx 2.8$, from which we estimate

$$
h \nu_{\text {peak }} \approx 15 \mathrm{eV} \times \alpha_{-1}^{0.36} S^{0.73} \lambda^{2.1} M_{6}^{-0.32}\left(\beta_{-1} \zeta\right)^{-0.45} \theta_{0.2}^{0.09}
$$

Long after the merger, the inner disk approaches a quasi-steady accretion track satisfying $3 \pi \nu \Sigma=$ $\dot{M_{\text {out }}}\left(1-\sqrt{R_{*} / R}\right)$, where $\dot{M_{\text {out }}}$ is a radially constant mass supply rate of the inner disk. The bolometric emission is brightest where the quantity $2 \pi R \nu \Sigma \Omega^{2}$ is maximal in the disk, which corresponds to $R=25 / 16 R_{*}$. Applying this to equation (3.5), we calculate the photospheric temperature and find the corresponding peak frequency for the monochromatic flux at very late times to be

$$
h \nu_{\text {peak }} \approx 0.71 \mathrm{keV} \times M_{6}^{-8 / 34}\left(\frac{\dot{M}}{\dot{M}_{\text {Edd }}} \frac{0.1}{\eta}\right)^{8 / 17}\left(\frac{R_{*} c^{2}}{3 G M}\right)^{-18 / 17}
$$

Above, the dependence on $S$ is replaced by a dependence on the mass supply rate, as at late times the disk loses memory of earlier accumulation near the inner edge. For our choice of $R_{*}=3 G M / c^{2}$, the radiative accretion efficiency is $\eta=1-\sqrt{1-2 G M /\left(R_{*} c^{2}\right)} \approx 0.12$ (Novikov \& Thorne 1973). The circumbinary disk is expected to reach this quasi-steady track after at least $t_{\mathrm{vis}, 0} \approx 120 M_{6}^{1.3} \mathrm{yr}$ has elapsed since merger. 
Figure 4 shows the spectrum of our fiducial model disk around an equal-mass, $10^{6} M_{\odot}$ binary as calculated at different times with respect to the merger, from decoupling time $(\approx 3 \mathrm{yr}$ before the merger) up to $120 \mathrm{yr}$ after the merger. We show the exact results using equation (3.7) in solid lines 5 , and the blackbody spectrum in dotted lines. In the figure, we only account for emission out to a radius of $1000 G M / c^{2}$, as we do not expect significant contribution of high-frequency photons from regions far outside $R_{0}$. We find that the spectrum of the circumbinary disk does not evolve significantly from that at decoupling until several hours before the merger, because the deepest parts of the central potential are not accessible to the circumbinary gas until that time. Our initial and final spectra agree qualitatively with those given in MP05. As with the bolometric light curve, however, significant evolution is ongoing well before and after the nominal estimate for the onset of the afterglow. Much of the evolution, however, is complete $\sim 10 M_{6}^{1.3} \mathrm{yr}$ after the merger.

\subsection{Possible Reprocessing of the X-ray Signature}

The frequencies calculated above suggest that an evolving afterglow at low redshift or low binary mass may be observable by existing and future X-ray and UV telescopes shortly after the GW event. MP05 discussed the possibility that the afterglow could be reprocessed to IR frequencies if the merged binary is enshrouded by gas and dust. Here we note that the circumbinary disk itself is a plausible candidate to reprocess the UV/X-ray afterglow to lower frequencies.

Ignoring general relativistic effects, the self-irradiating flux of the disk with viscously dissipated flux $F_{\text {visc }}$ can be written (see, e.g., Blaes 2004)

$$
F_{\text {irr }}(R)=\frac{1-A}{4 \pi R^{2}} \int 2 \pi R^{\prime} \sin \vartheta F_{\text {visc }}\left(R^{\prime}\right) d R^{\prime}
$$

where $A$ is the albedo of the disk and $\vartheta$ is the angle between the disk surface and the irradiating light ray. In our disk solutions, most of the dissipated disk luminosity $L_{\mathrm{visc}}$ is produced at small radii due to the fact that the viscously dissipated flux is proportional to $\Omega^{2}$. Thus, if $R$ is much greater than the size of the central emitting region then it is a reasonable approximation to replace the integral in the above equation with $L_{\text {visc }} \sin \vartheta$. If one further assumes that $A$ is small and that the irradiating flux is large compared to the local value of $F_{\mathrm{visc}}$, the luminosity reprocessed by the outer disk will be $L_{\text {irr }} \lesssim L_{\text {visc }} \sin \vartheta$. The light-travel time is short ( $\sim 10^{-2} M_{6}$ yr across distances $\sim 10^{5} G M / c^{2}$ ) compared to the timescale for viscous evolution of the inner disk, and thus we expect the reprocessing processes below to be effectively instantaneous.

\footnotetext{
${ }^{5}$ The function $\nu F_{\nu}$ peaks at $\xi=3.9$, so replace the leading factor $15 \mathrm{eV}$ in equation (3.9) above with $21 \mathrm{eV}$ to derive the locations of the peaks in the plots of Figure 4
} 
One way that the factor $\sin \vartheta$ can be large is if there is a warp in the disk that offsets the planes of the outer and the innermost regions. The circumbinary disk is expected to be aligned with the binary orbit in the binary's vicinity, where tidal torques dominate viscous torques. Farther out in the disk, the disk may have a quasi-steady warp with respect to the binary (Ivanov et al. 1999). Because the warp would dissipate on timescales longer than the afterglow itself, it could act to promptly reprocess the afterglow into longer wavelengths.

We also find that the evolution of the innermost accretion flow itself could effect a disk geometry that is conducive to reprocessing. If there is no warp and the emitting region is in the equatorial plane, then (e.g. Blaes 2004)

$$
\sin \vartheta \sim \frac{H}{R}\left(\frac{d \ln H}{d \ln R}-1\right) .
$$

As we show below (Figures 5 and 7), just outside the regions of brightest emission $\left(R \lesssim R_{0}\right)$ and around the time of merger our disk solutions are often moderately geometrically thick $(H / R \gtrsim 0.1)$ and steeply flared geometrically (large positive $d \ln H / d \ln R$ ). That is, the circumbinary disk itself may serve as a shroud of gas that can capture and reprocess the high-frequency accretion signature.

Either of the two effects considered above could cause the luminous inner disk around the binary remnant to irradiate the outer disk at a sufficiently large angle $\vartheta$ to effect a reprocessed IR/optical signature of substantial luminosity.

\subsection{Possible Effects of Advection and Super-Eddington Winds}

Above, we largely confirmed the findings of MP05, with the additional suggestion that accretion afterglows of MBH mergers may be observable somewhat earlier than estimated in that study. However, we also find that at late times the spreading accretion flow becomes geometrically thick near the $\mathrm{MBH}$ remnant, with the scale-height-to-radius ratio $H / R$ formally exceeding unity. Here, $H=\Sigma / \rho=c_{\mathrm{s}} / \Omega$, where $c_{\mathrm{s}}$ is the sound speed $\sqrt{\gamma P / \rho}$ and $\gamma \approx 4 / 3$ is the adiabatic index for a radiation-pressure-dominated gas. The radial profile of $\rho$ is calculated from the disk temperature profile, which is obtained through equations (3.5) and (3.8) and the total pressure in the disk $P_{\text {gas }}(\rho, T)+P_{\text {rad }}(T)$.

For some binary and disk parameters, the inner edge of the disk may already be geometrically thick at decoupling (MP05; see Table 1). In such a scenario, the circumbinary gas may be free to accrete off-binary plane, and the inner cavity may not be as evacuated as we have assumed here, and the difference between the pre- and post-merger disk spectra may be less easily discernible. In addition, after the merger, it is also possible for the accreting gas to become radiatively inefficient, 
as in the advection-dominated accretion flow (ADAF; Narayan \& Yi 1994; Abramowicz et al. 1988) model, which would make it more difficult to observe].

As argued in MP05, if horizontal advection is competitive with viscous dissipation then the physical disk would be thinner than suggested by our simple estimate of the ratio $H / R$, as in the "slim disk" model of Abramowicz et al. (1988). In this paper, we do not attempt to develop a self-consistent time-dependent model that incorporates vertical disk structure and radial advection. Rather, we proceed below as if the disk evolution can be approximated by the thin-disk evolution described by equation (2.6) while keeping the above caveats in mind.

The absolute value of the advected flux can be estimated as (e.g., Abramowicz et al. 1988)

$$
\mathcal{Q}_{\mathrm{adv}}=\frac{\dot{M}}{\pi R^{2}} \frac{P_{\mathrm{rad}}}{\rho}\left|\frac{d \ln \rho}{d \ln R}-3 \frac{d \ln T}{d \ln R}\right| .
$$

Substituting equation (2.4) for the local accretion rate and equation (A18) for the radiated flux, we crudely estimate the advected to radiated flux ratio as:

$$
\frac{\mathcal{Q}_{\mathrm{adv}}}{\mathcal{Q}_{\mathrm{rad}}} \approx \frac{4}{3} \frac{P_{\mathrm{rad},} H}{\Sigma R^{2} \Omega^{2}}\left[1+\frac{d \ln (\nu \Sigma)}{d \ln R}\right]\left|\frac{d \ln \rho}{d \ln R}-3 \frac{d \ln T}{d \ln R}\right|
$$

The simple calculations of the evolving disk spectrum performed in $\$ 3.2$ are not highly dependent on the disk temperature (our Figure 4 agrees very well with the spectra shown in MP05, despite the fact that we have calculated a somewhat lower value of $T_{0}$ ). However, the structure of the disk at the inner edge at decoupling and the subsequent evolution of the accretion flow is likely to depend much more sensitively on the temperature profile. In the radiation-dominated limit, $H$ is proportional to $P_{\mathrm{rad}} \Sigma^{-1} \Omega^{-2}$ so from equation (3.13) we see that the advected flux is roughly proportional to $\nu T^{8} \Sigma^{-1} \Omega^{-2} R^{-2}$. This implies that near the inner edge, $\mathcal{Q}_{\text {adv }} \propto T^{62 / 7}$ approximately (see Appendix $\mathrm{A}$ for how disk quantities such as $\Omega$ scale with the temperature near the edge). Thus, we expect the possible transition of the disk from a thin disk to an advective/slim disk or a geometrically thick ADAF - and the corresponding spectral evolution - to be a delicate function of the thermal structure of the disk at decoupling. For example, MP05 estimated a midplane temperature at the inner edge that was $\sim 1.3$ times higher than we did, with most of the discrepancy due to the aforementioned factor of two in the flux formalization. Although this does not result in significant differences between our values and theirs for the decoupling radius or the relevant timescales, it does lead those authors to estimate that the advective flux is more significant at the disk edge at decoupling than we do. We estimate that immediately outside the disk edge,

$$
\frac{\mathcal{Q}_{\mathrm{adv}}}{\mathcal{Q}_{\mathrm{rad}}} \gtrsim 10^{-2} \alpha_{-1}^{1.5} S^{4.9} \lambda^{-7.6} M_{6}^{-0.24}\left(\beta_{-1} \zeta\right)^{-1.9} \theta_{0.2}^{2.4}
$$

\footnotetext{
${ }^{6}$ Here, we mean a single-temperature, collisional, radiatively inefficient flow (as opposed to the collisionless, optically thin, two-temperature models in the literature, e.g., Shapiro et al. 1976; Rees et al. 1982; Narayan \& Yi 1995).
} 
where MP05 estimated $\mathcal{Q}_{\text {adv }} / \mathcal{Q}_{\text {rad }} \sim 0.44$. The flux ratio is higher at the edge due to the steep temperature and density gradients there. The equation above suggests that it is highly sensitive to all of the disk and binary parameters save for the binary mass.

In Figure 5 we show the radial profiles of several additional disk quantities for the same parameters, profile and times as shown in Figure 1. Along with the surface density, we also plot: the simple estimate from equation (3.14) for the advected-to-radiated flux ratio, $\mathcal{Q}_{\text {adv }} / \mathcal{Q}_{\text {rad }}$; the estimated scale-height-to-radius ratio $H / R$; and $F / F_{\text {Edd }}$, the ratio of the locally dissipated flux to $F_{\text {Edd }} \approx H \Omega^{2} c / \kappa_{\text {es }}$, the local Eddington flux limit associated with the vertical component of gravity. For the last ratio, we find

$$
\frac{F}{F_{\text {Edd }}} \approx 0.06 \frac{\Sigma(R, t)}{\Sigma_{0}} \sqrt{1+(H / R)^{-2}} r^{n-1} \alpha_{-1}^{0.34} S^{1.2} \lambda^{-1.70} M_{6}^{-0.08}\left(\beta_{-1} \zeta\right)^{-0.42} \theta_{0.2}^{0.08} .
$$

Figure 5 confirms that for fiducial parameters, the circumbinary accretion flow remains locally subEddington. For fiducial parameter values, we find that a relatively high surface density $\Sigma / \Sigma_{0} \gtrsim$ 1 (or large $S$ ) is required to reach a super-Eddington flux near $R=R_{*}$, and the disk is thus not necessarily likely to develop super-Eddington winds at levels which would significantly affect the light curves or spectra presented in Figures 3 and 4 . At late times, however, the nominal advective flux exceeds the locally dissipated flux as the disk becomes geometrically thick, which may lead the disk to become radiatively inefficient.

This hypothetical transition of the innermost accretion flow from a thin disk to a geometrically thick flow could act to suppress the X-ray afterglow. We estimate very simply the effect of advection on the emitted spectrum as follows. We assume that the emitted flux is suppressed by a factor $f \equiv \max \left\{0,1-\mathcal{Q}_{\text {adv }} / \mathcal{Q}_{\text {rad }}\right\} \leq 1$, and that the advected energy is not re-emitted. We also assume that advection acts to suppress emission evenly across all photon frequencies. The resulting advection-limited spectrum is shown in Figure 6 alongside a spectrum calculated without accounting for advection (i.e. the same disk spectra as those shown in Figure 4). from decoupling to 20 years after the merger. We compare the two classes of spectra at decoupling, and 1 month, $2 \mathrm{yr}, 5 \mathrm{yr}, 9.2 \mathrm{yr}$ and $20 \mathrm{yr}$ after the merger, with thicker lines in the figure denoting later times. If the disk were to remain radiatively efficient at all radii and times, the emission at high frequencies would steadily increase until the quasi-steady thin-disk track is reached. If instead the innermost disk becomes advective, this suppresses the high-energy emission at late times. Our results indicate that our fiducial disk model may become increasingly less bright as the inner disk becomes increasingly geometrically thick. However, the calculations shown in Figure 6 suggest that a brightening afterglow may still be visible in the soft X-ray for a short time $\sim 10^{-2} t_{\text {visc }, 0}$ after the merger. This suggests that the circumbinary gas may have two observable signatures of interest: an initial brightening phase with a soft X-ray afterglow, and a subsequent dimming phase as it transitions into an ADAF. 
To further investigate the possible consequences of advection on the circumbinary disk evolution, we turn to a different disk model whose inner edge is already geometrically thick at decoupling. We take $S=5$ and $\zeta=1 / 3$ (binary mass ratio $q \approx 1 / 10$ ), keeping all other parameters the same as in the fiducial model. The new choices for $S$ and $\zeta$ are physically plausible ones. If the mass supply rate of the outer disk is comparable to the Eddington limit of the central binary, we can expect $S>1$ at decoupling as a result of mass accumulation. We may also expect $q \sim 1 / 10$ mergers to be more common 7 than near-equal-mass mergers because merging galaxies (dark matter halos) have unequal masses and because the mass contrast between the MBHs is expected to be higher than that of the host halos (i.e. the observationally inferred MBH-to-host-halo mass ratio relation is a steeper-than-linear function of the halo mass; Ferrarese 2002). Cosmological merger tree calculations for MBH mergers consistently show that contributions to the LISA data stream from MBH coalescences will be dominated by moderately low-q events (e.g., Volonteri et al. 2003; Sesana et al. 2007; Tanaka \& Haiman 2009).

With this new set of parameters, the disk decouples at a time $t_{\mathrm{GW}, 0} / 4 \approx 0.30 \mathrm{yr}$ before the merger, when the binary has reached a semimajor axis $a_{0} \approx 54 G M / c^{2}$. The nominal estimate for the onset of the afterglow emission is $t_{\mathrm{EM}} \approx 0.91 \mathrm{yr}$ after the merger. The inner edge of the disk has a somewhat higher temperature, $T_{0} \approx 8.7 \times 10^{6} \mathrm{~K}$. We again use the initial density profile given by equation (2.7). In Figure 7 we show the same disk quantities for this thick disk as shown for the fiducial disk in Figure 5. This time, the disk remains geometrically thick and radiatively inefficient inside the initial edge radius throughout its evolution. As we did for the fiducial disk model, we estimate the advective suppression of the spectrum for the thick disk and show results in Figure 8 , This simple calculation suggests that a disk that is sufficiently geometrically thick at decoupling may not, in principle, exhibit any observable spectral evolution.

On the other hand, we also find that the viscously dissipated flux of this thick circumbinary disk becomes super-Eddington at late times (Figure 8d). Begelman (2002) proposed that accretion disks with a super-Eddington flux may be able to stay geometrically thin and radiatively efficient. Such accretion flows may also generate powerful outflows and flares. While our simple models do not self-consistently treat advection or the super-Eddington regime, our results underscore the need for further study of this class of accreting systems.

\footnotetext{
${ }^{7}$ Provided that they can overcome the "last-parsec problem."
} 


\section{Conclusion}

We have presented a simple semianalytic model for the viscous evolution of a thin circumbinary disk around a MBH binary, in the final stages of the binary's evolution. Using this model, we have estimated the time dependence of the thermal spectrum immediately after the binary merger. In what may be the most optimistic scenario, a rapidly evolving soft X-ray signature may be observed soon after the GW event, perhaps years earlier than previously estimated. It is worth emphasizing that the bolometric luminosity is several orders of magnitude brighter than many of the EM counterpart candidates proposed in the literature, and in extreme cases may exceed the Eddington luminosity of the central MBH. An important feature of the afterglow mechanism discussed in this paper is that it is not strongly limited by the mass of the circumbinary disk. This is in stark contrast to recoil-powered afterglows whose luminosities and observational prospects are generally limited by the mass of the gas bound to the recoiling remnant (e.g., Bode \& Phinney 2007; Schnittman \& Krolik 2008; Lippai et al. 2008; Shields \& Bonning 2008; O’Neill et al. 2009; Megevand et al. 2009; Corrales et al. 2009; Rossi et al. 2009; Anderson et al. 2009).

The reason why an afterglow may be observable so early is because the power per unit area dissipated by viscous dissipation scales as $F \propto \nu \Sigma \Omega^{2}$. The potential is sufficiently deep close to the $\mathrm{MBH}$ remnant that in this region even low surface densities can generate more power than elsewhere in the disk. A central conclusion of this paper is that enough gas may be able to viscously follow the binary to merger. The presence of gas in the vicinity of the binary at merger may have significant implications for other proposed EM counterpart mechanisms, as well. Some of this gas can reasonably be expected to contribute to circumprimary or circumsecondary accretion disks which could generate additional observable signatures even if the total gas mass captured in this way is small (Armitage \& Natarajan 2005; Lodato et al. 2009; Chang et al. 2009).

In addition to the possibility that the accretion afterglow proposed by MP05 may be observable earlier than previously estimated, our results raise interesting possibilities about the observable features of the post-merger disk at later times. Most of the luminosity is generated in the innermost disk $\left(R \lesssim 20 G M / c^{2}\right)$, and may be partially reprocessed to IR/optical frequencies by the outer disk $\left(R \gtrsim 100 G M / c^{2}\right)$. We have considered two mechanisms that could reasonably reprocess a significant fraction of the inner-disk emission: a geometrical warp in the disk just outside the emitting region; and the steep geometrical flaring of the vertical thickness of the inner disk as it evolves We find that the bolometric luminosity of the time-dependent afterglow can exceed the Eddington luminosity of the binary, without violating the local Eddington flux limit. We conclude that the EM signature - the unobstructed signature in soft X-rays, as well as the reprocessed signature in IR/optical - could become comparable to the Eddington luminosity of the binary.

Our calculations also suggest that as it spreads, the innermost disk may become geometrically thick and thus radiatively inefficient, even if it is neither geometrically thick nor radiatively inef- 
ficient at decoupling. If the disk is initially geometrically thin at decoupling, then it may emit an afterglow that is powered by the newly formed deep central potential before becoming geometrically thick near the remnant at later times. In such a scenario, an accretion disk around a recently merged MBH remnant may provide a unique system where the transition from a radiatively efficient to an inefficient accretion state can be monitored on humanly tractable timescales.

In the most pessimistic scenario, it seems possible for the disk to become geometrically thick even before the binary merges. In such a situation a disk could behave like an ADAF and it is unclear whether a circumbinary cavity should exist at all. Even if a cavity were kept open until decoupling, advective losses may suppress any observable spectral evolution for the disk emission. As suggested by MP05, horizontal advection could also act to make the disk thinner than we have considered here, as in the "slim disk" models of Abramowicz et al. (1988). A slim disk would remain somewhat radiatively efficient and thus could still exhibit an observable evolution of the spectrum or luminosity. Another possible mechanism to keep the circumbinary disk radiatively efficient is super-Eddington fluxes (Begelman 2002). We find that in the same regions of the parameter space where the disk becomes very geometrically thick, the viscously dissipated flux also becomes very high. The nominally radiated fluxes near the central regions around the $\mathrm{MBH}$ remnant can be super-Eddington for physically reasonable parameter values, and this could also help the disk produce an observable evolving EM signature.

Advection-dominated accretion or a super-Eddington flow may produce powerful outflows (e.g., Blandford \& Begelman 1999). The outright viscous dissipation of super-Eddington fluxes near the center could also result in a strong outflow. The spin of the MBH remnant and its orientation are likely to be well constrained from the preceding LISA observation of the merger GWs. As such, the observation of a jet near a recently merged $\mathrm{MBH}$ binary would present an unprecedented opportunity to study a MBH-powered jet where the spin of the central engine is precisely and independently constrained.

We conclude that the observation of the EM accretion afterglow of a $\mathrm{MBH}$ merger is likely to provide a windfall of empirical constraints on the physics of gas accretion onto MBHs. Such an observational opportunity would be unprecedented on three points. First, the "initial condition" for the structure of the evolving flow may be relatively well characterized thanks to models for circumbinary disks and the theoretical understanding of the orbital evolution of the binary leading up to the merger. Second, the accretion flow is likely to evolve on humanly tractable timescales. Such an accretion signature will act as a probe of the viscosity in the flow, in contrast with a steady $\alpha$-disk in which the emission is independent of the $\alpha$ parameter. Third, the mass, spin and orientation of the central MBH will have been independently determined by LISA. The last point is important in terms of emission geometry, energetics, and particularly significant if the accretion flow fuels an outflow or a jet as it fills the central cavity. 
In this work, we have used idealized models with a simple radial power-law prescription for the gas viscosity. However, in the $\alpha$-viscosity model the viscosity is also a function of surface density and disk thickness. In light of the large range of $\Sigma$ and $H / R$ values found in the regimes of interest in our solutions, it would not be surprising if a more realistic viscosity prescription led to significantly different results from those presented here. Even in our highly idealized calculations, the richness of the physical problem that these systems represent is readily apparent. Given advection, radiation-pressure dominance, super-Eddington fluxes, issues of disk stability and geometry, and rapid viscous evolution, the circumbinary accretion flows around a merging MBH binary are likely to produce intriguing observational signatures that would serve as unprecedented probes of fundamental astrophysical processes. The interesting possibilities raised by our simple models underscore the need for more detailed investigations of this interesting class of objects.

We thank Zoltán Haiman for insightful conversations and constructive comments on the manuscript. We are grateful to the anonymous referee for comments that helped improve this paper. This work was supported by NASA ATFP grant NNXO8AH35G. 


\section{APPENDICES}

\section{A. Properties of the Circumbinary Disk After Decoupling}

We review below the properties of the inner circumbinary disk at the time of decoupling. Decoupling is defined as the time when $t_{\mathrm{GW}}$, the timescale on which the binary orbit shrinks, becomes shorter than $\beta t_{\text {visc }}$, the timescale on which the central cavity refills due to viscous spreading of the disk. The procedure is as follows. First, the decoupling condition $t_{\mathrm{GW}} \sim \beta t_{\text {visc }}$ relates the gas properties at the inner edge to the orbital parameters of the binary in terms of the disk midplane temperature. Second, the midplane temperature is calculated by making simple assumptions about the thermal structure of the disk. MP05 performed these calculations for the inner edge of the disk; we follow their approach closely and reproduce their results while pointing out a few differences. We also discuss possible ways to set the radial power $n$ of the prescribed viscosity in our time-dependent solutions.

Assumptions underlying the model are detailed in the body of this paper and in MP05. Notation for physical quantities is given in our $\$ 2$. Fiducial parameter values are $M=10^{6} M_{\odot} M_{6}$, $\zeta \equiv 4 q /(1+q)^{2}=1, \alpha=0.1 \alpha_{-1}, \beta=0.1 \beta_{-1}, \lambda=1, S=1$, and $\theta_{0.2}=1$. Unless otherwise specified, the subscript " 0 " refers to the value at decoupling when applied to binary properties; when applied to gas properties, it refers to the value at decoupling time and at the inner edge of the disk.

\section{A1. Properties at decoupling as functions of midplane temperature}

For circular orbits with semimajor axis $a$, the timescale of gravitational-wave-driven decay of the binary separation is given by:

$$
t_{\mathrm{GW}} \equiv \frac{a}{d a / d t}=\frac{5}{16} \frac{c^{5}}{G^{3} M^{3}} a^{4} \zeta^{-1} .
$$

The viscous time is

$$
t_{\mathrm{visc}}=\frac{2}{3} \frac{R^{2}}{\nu}=\frac{R^{2} \Omega \mu m_{\mathrm{p}}}{\alpha k T} \approx 144 \mathrm{yr} \times \alpha_{-1}^{-1} \lambda^{1 / 2} T_{6}^{-1}\left(\frac{a}{100 G M / c^{2}}\right)^{1 / 2},
$$

where $T=10^{6} \mathrm{~K} T_{6}$ is the midplane temperature.

Applying the decoupling condition $t_{\mathrm{GW}, 0} \sim \beta t_{\mathrm{visc}, 0}$ gives the following quantities in terms of 
Table 1. Inner Circumbinary Disk at Decoupling ${ }^{\mathrm{a}}$

\begin{tabular}{cccccccc}
\hline \hline Variable & Factor & $\alpha_{-1}$ & $S$ & $\lambda$ & $M_{6}$ & $\beta_{-1}, \zeta$ & $\theta_{0.2}$ \\
\hline$a_{0} /\left(G M / c^{2}\right)$ & 126 & -0.34 & -0.24 & 0.70 & 0.08 & 0.42 & -0.08 \\
$t_{\mathrm{EM}}(\mathrm{yr})$ & 9.2 & -1.36 & -0.98 & 2.80 & 1.32 & $1.7,0.7$ & -0.34 \\
$\Sigma_{0}\left(\mathrm{~g} \mathrm{~cm}^{-2}\right)$ & $6.2 \times 10^{5}$ & -0.68 & 0.51 & -0.60 & 0.16 & -0.15 & -0.17 \\
$T_{0}\left(10^{6} \mathrm{~K}\right)$ & 1.3 & 0.19 & 0.86 & -1.95 & -0.28 & -0.49 & 0.30 \\
$H_{0} / R_{0}$ & 0.17 & 0.76 & 2.43 & -3.80 & -0.12 & -0.95 & 1.19 \\
$P_{\text {rad }, 0} / P_{\text {gas }, 0}$ & 430 & 1.67 & 4.25 & -7.35 & -0.04 & -1.84 & 2.17 \\
$\mathcal{Q}_{\text {adv }, 0} / \mathcal{Q}_{\text {rad }, 0}$ & $2.0 \times 10^{-2}$ & 1.52 & 4.86 & -7.60 & -0.24 & -1.90 & 2.38 \\
\hline
\end{tabular}

${ }^{a}$ At decoupling, the variable in column 1 equals the factor in column 2 multiplied by the column head parameters raised to the powers indicated in columns 3-8. All quantities except for $a_{0}$ are evaluated at the inner edge of the circumbinary disk. We derive power-law dependencies identical to those found by MP05. Note, however, that we derive a somewhat lower temperature than MP05, and estimate that the disk is significantly less geometrically thick than they did. See Appendix A for detailed calculations. 


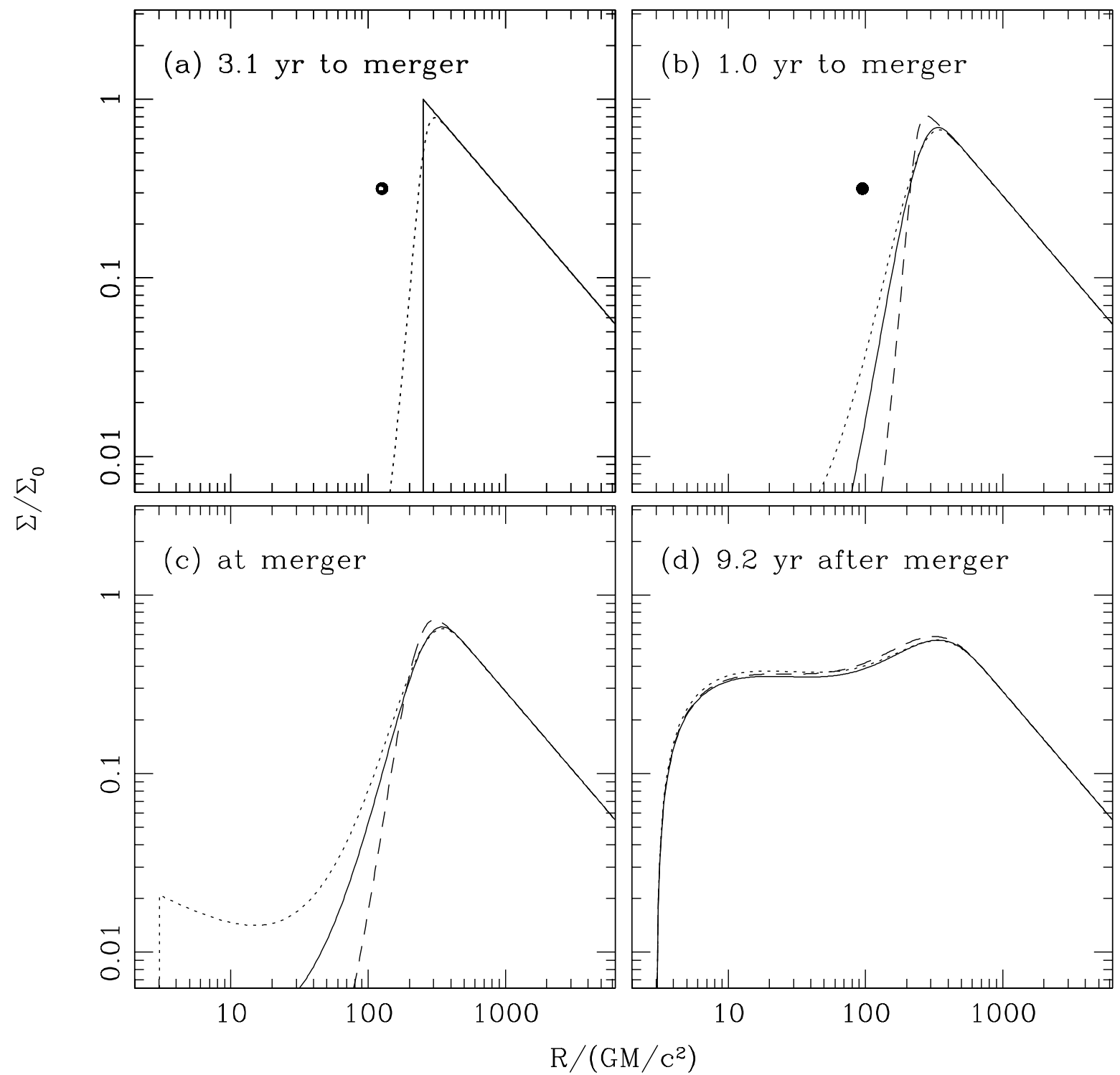

Fig. 1.- Snapshots of the time-dependent solutions to equation (2.5) for the surface density $\Sigma / \Sigma_{0}$ for fiducial disk parameters. We evolve the solution by applying (2.6) with $n=0.4$, which is roughly consistent with the physical prescription $\nu \propto T / \Omega$ at late times and small radii, when the disk approaches a quasi-steady accretion track satisfying $\Sigma \nu \propto\left(1-\sqrt{R_{*} / R}\right)$. Times in the figure are scaled to the merger of an equal-mass, $10^{6} M_{\odot}$ binary. Panel (a) shows the initial surface density profiles at decoupling. Panels (b), (c) and (d) show the evolved profiles at 1 year before the merger, at merger, and 9.2 years after the merger, respectively. We also evolve a third profile (dashed lines) that qualitatively describes a scenario where the binary continues to open a gap even after the nominal decoupling condition (see text for details). In panels (a) and (b), the semimajor axis of the binary is shown schematically with black circles (the orbit shrinks by $\approx 25 \%$ between the panels). After the merger, we impose a zero-torque condition at $R_{*}=3 G M / c^{2}$; surface density profiles at merger (Panel c) are truncated at this radius and subsequently evolved with the new boundary 


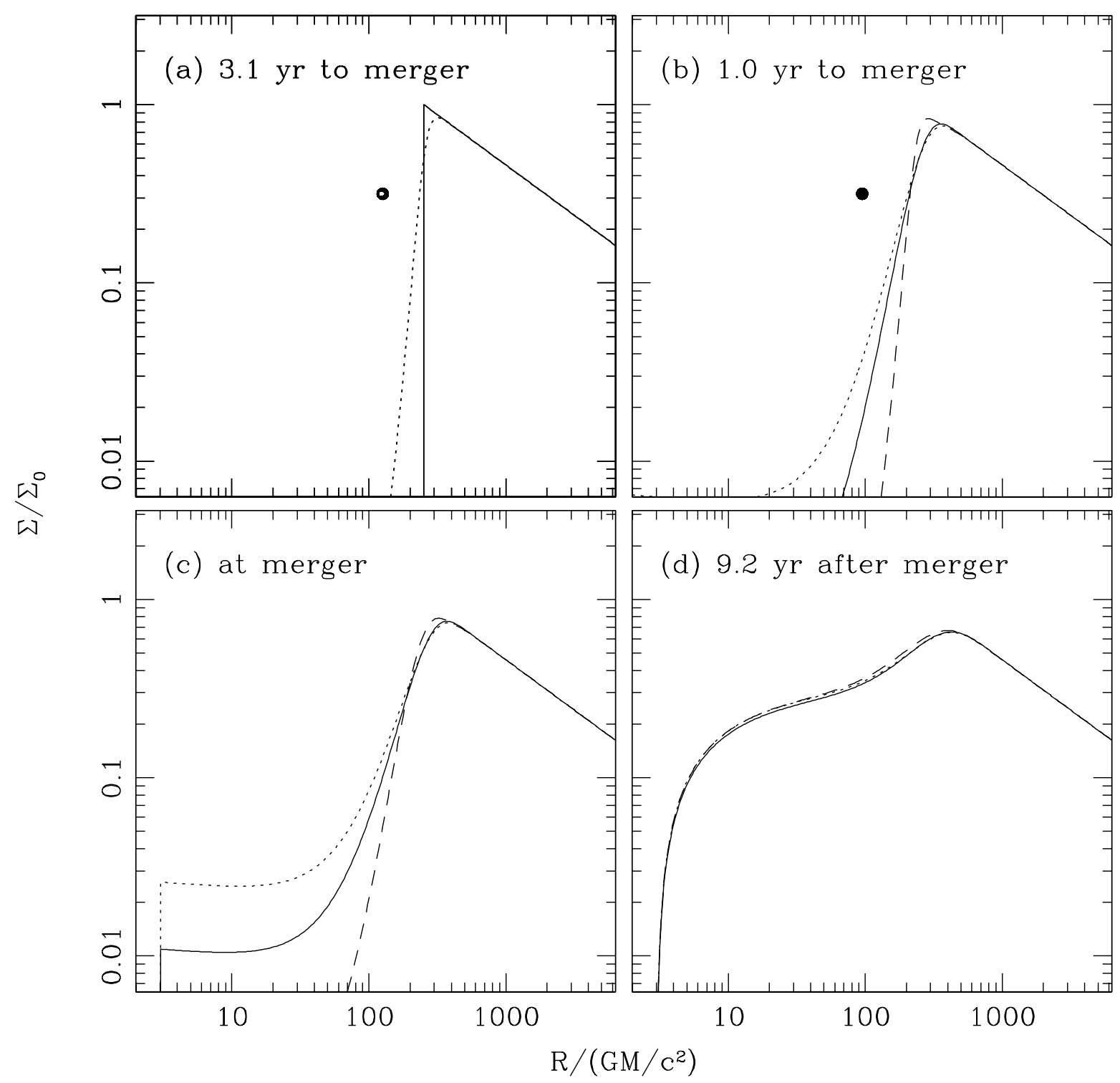

Fig. 2.- Same as Figure 1, but with $n=11 / 170 \approx 0.065$, which is the value consistent with a physical prescription for viscosity, $\nu \propto T / \Omega$, just outside the inner edge of the disk. We find that our qualitative findings are insensitive to the value of $n$, as long as the quantity $2-n$ is not much smaller than unity. 


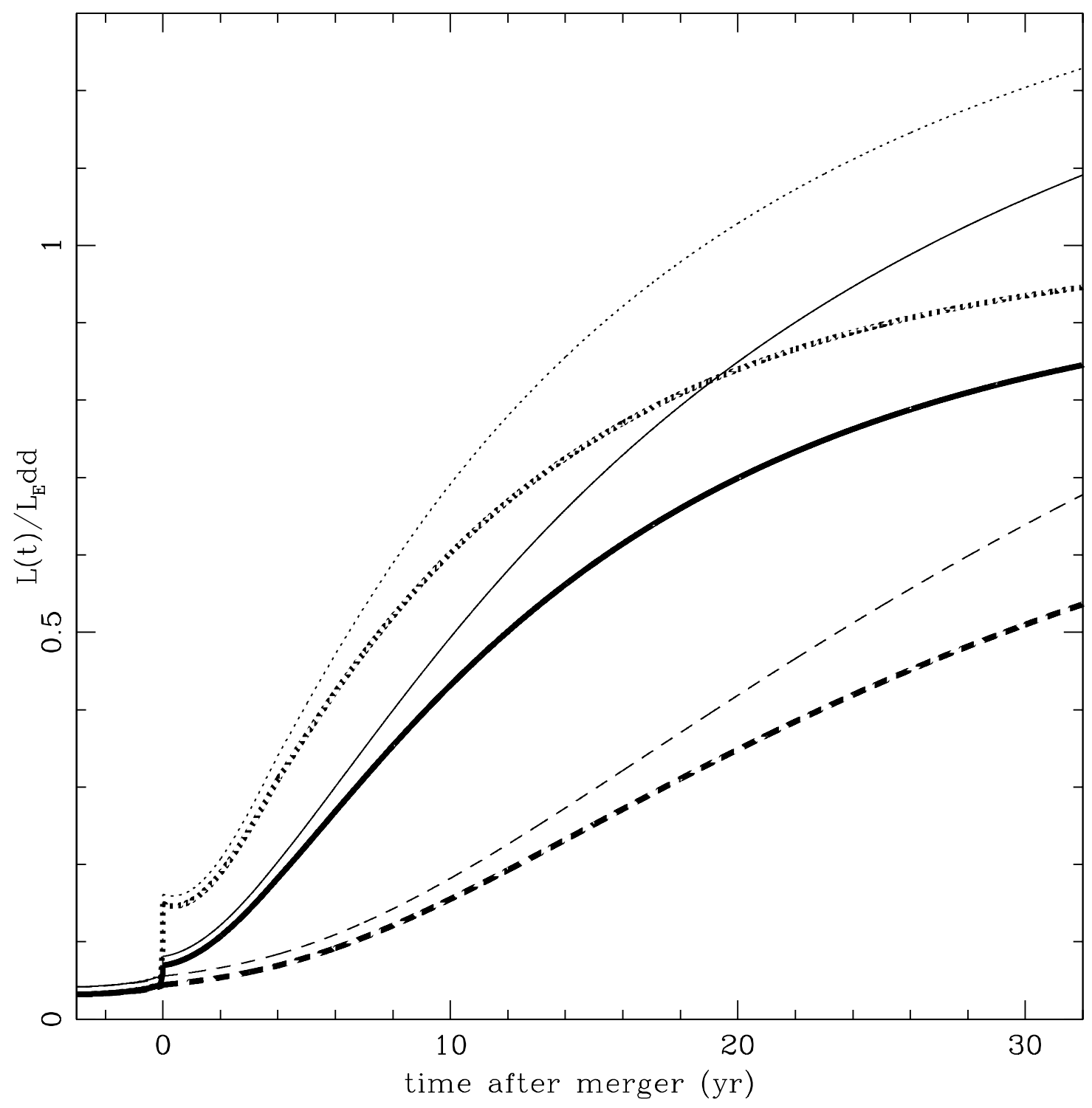

Fig. 3. - The viscously dissipated bolometric disk luminosity for fiducial parameters, in units of the Eddington luminosity. The time is relative to merger. The thick lines depict the light curve for density profiles that have a decretion power-law $m=\partial \ln (\nu \Sigma) / \partial \ln R=-1 / 2$ outside the inner edge. The thin lines are for profiles with $m=0$ outside the inner edge. The light curves are somewhat sensitive to the value of $n \equiv \partial \ln \nu / \partial \ln R$, but have the same qualitative behavior. The light curves shown here correspond to disks with viscosity index $n=0.4$ (solid lines), $n=11 / 170$ (dashed lines) and $n=1$ (dotted lines). Our model predicts significant evolution before and after $t_{\mathrm{EM}} \sim 9 M_{6}^{1.3} \mathrm{yr}$, our re-evaluation of the nominal time proposed by MP05 for the onset of the viscosity-powered afterglow. The sharp increase in luminosity at merger time is a somewhat artificial effect that arises from the fact that we account only for the flux dissipated outside the radius $2 \lambda a(t)$ (see text), which shrinks rapidly in the days before merger. Note that the luminosity scales with the massaccumulation parameter $S$ as $L \propto S^{1.2}$, and so could be significantly enhanced if the inner disk is sionificantlv more massive than a comnarable steadv-state Shakura-Sunvaev disk 


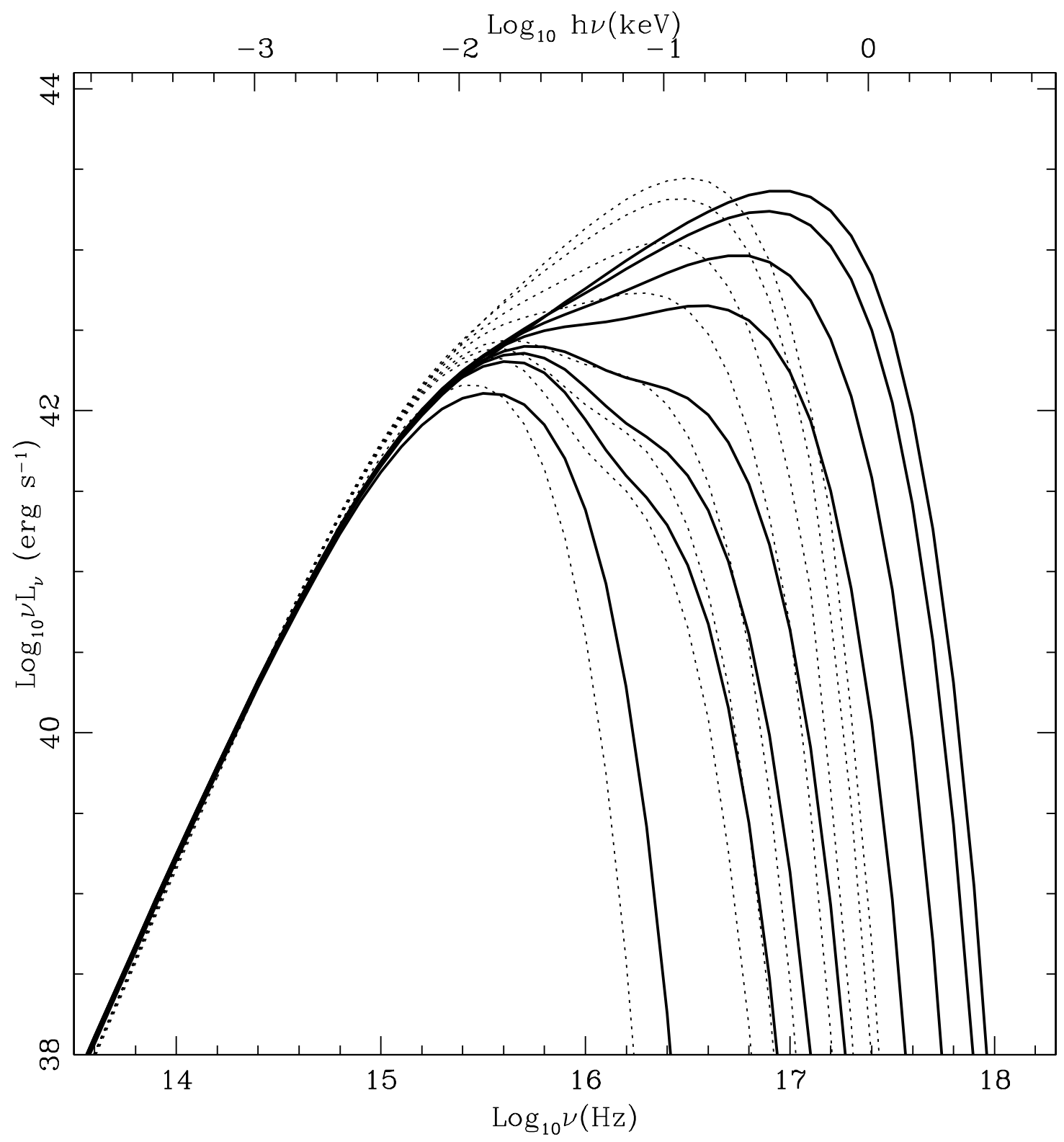

Fig. 4.- The thermal disk spectra emitted at (from left to right): decoupling (3.1 years before the merger), 1 month after, 1 year after, 2 years after, 5 years after, 9.2 years after, 20 years after, and 120 years after the merger. The thick, solid lines show the spectra calculated using the "graybody" formulation in equation (3.7), while the thin, dotted lines show the spectra calculated assuming the disk instead emits as a perfect blackbody. For any given snapshot, the two sets of lines show different spectral distributions of the same bolometric luminosity. For the gray-body case, the 9.2year snapshot corresponds to our re-evaluation of the nominal estimate by MP05 for the onset of the $\mathrm{X}$-ray afterglow, and 120 years is the viscous time at the disk edge at decoupling. 

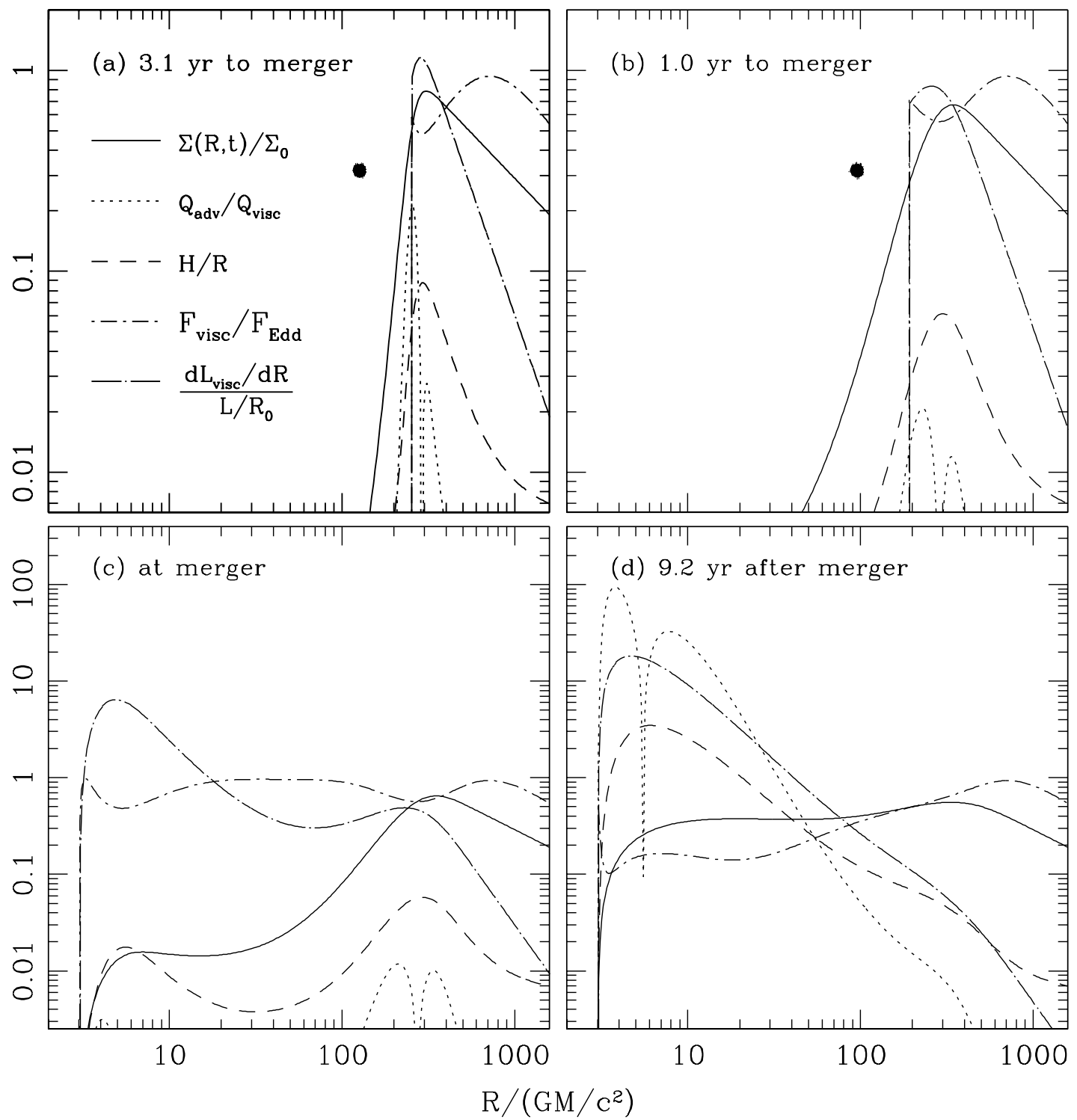

Fig. 5.- For our fiducial disk model: the surface density, scale-height-to-radius ratio $H / R$; estimated ratio of the advected to radiated flux $\mathcal{Q}_{\text {adv }} / \mathcal{Q}_{\text {rad }}$; the ratio of the local flux to the Eddington limit $F_{\text {visc }} / F_{\text {Edd }}$; and the flux contribution $d L_{\text {visc }} / d R=2 \pi R F_{\text {visc }}$ normalized to $L / R_{0}$. The disk is geometrically thin and advection is largely insignificant until the merger. After the merger, however, the inner disk becomes formally geometrically thick and advection-dominated (Panel d). Note that in panels (c) and (d) the vertical scale is different from the other two panels. The steep dips in $\mathcal{Q}_{\text {adv }} / \mathcal{Q}_{\text {rad }}$ are due to the advected flux changing sign (see equation [3.14]). 


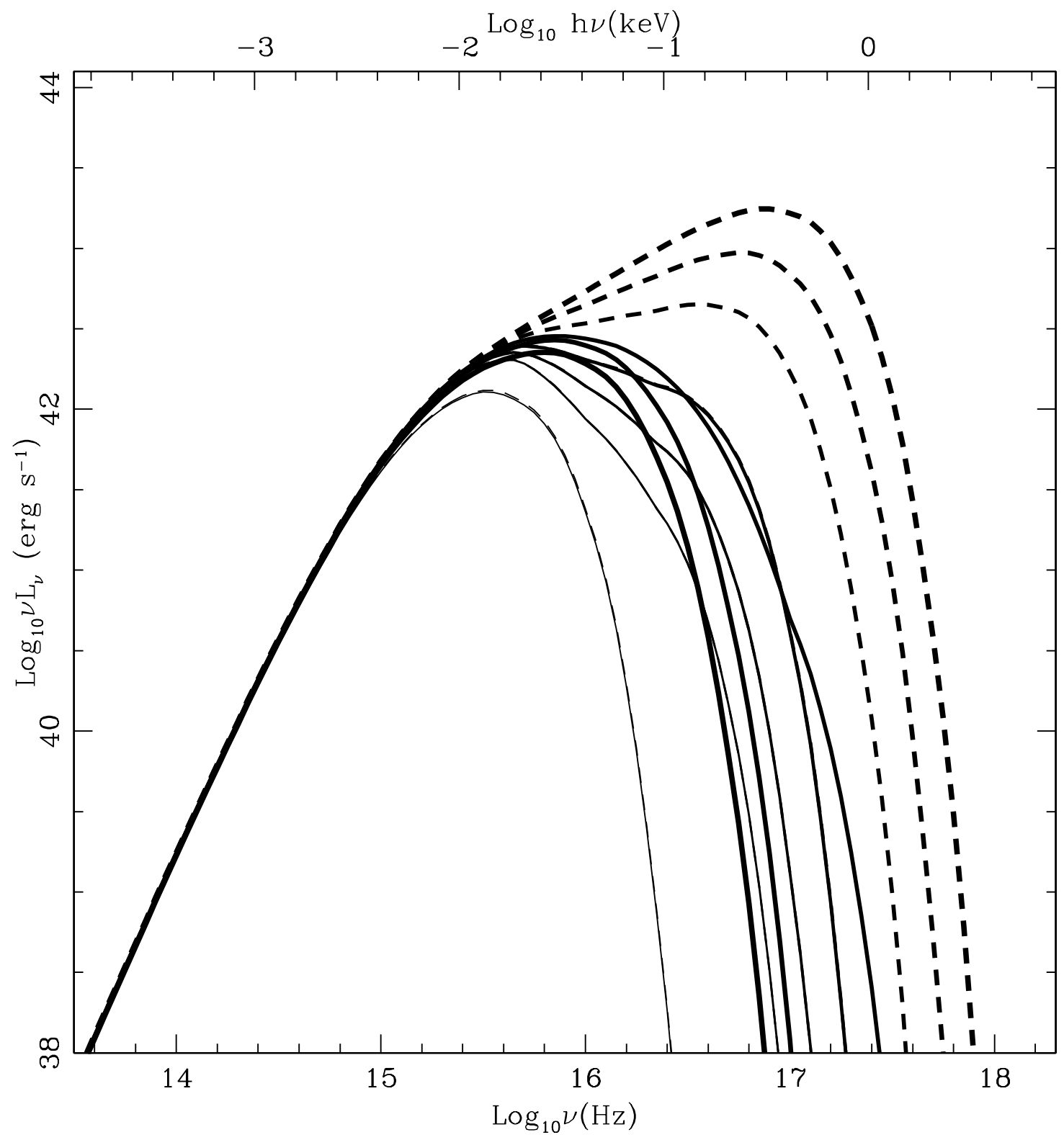

Fig. 6.- Emission spectra for the fiducial circumbinary disk. The dashed lines show the same spectra as calculated in Figure 4 from thinnest to thickest curves, at decoupling and at 1 month, 2 $\mathrm{yr}, 5 \mathrm{yr}, 9.2 \mathrm{yr}$ and $20 \mathrm{yr}$ after the merger. The solid lines show the spectra at the same times, but after subtracting the estimated advected flux. The disk is initially geometrically thin and advection is not significant until after the merger. At late times, the inner disk becomes geometrically thick and radiatively inefficient, and this could lead to reduced overall X-ray emission. The disk emits briefly emits soft X-rays at intermediate times, $\sim 2 \mathrm{yr}$ after the merger. 


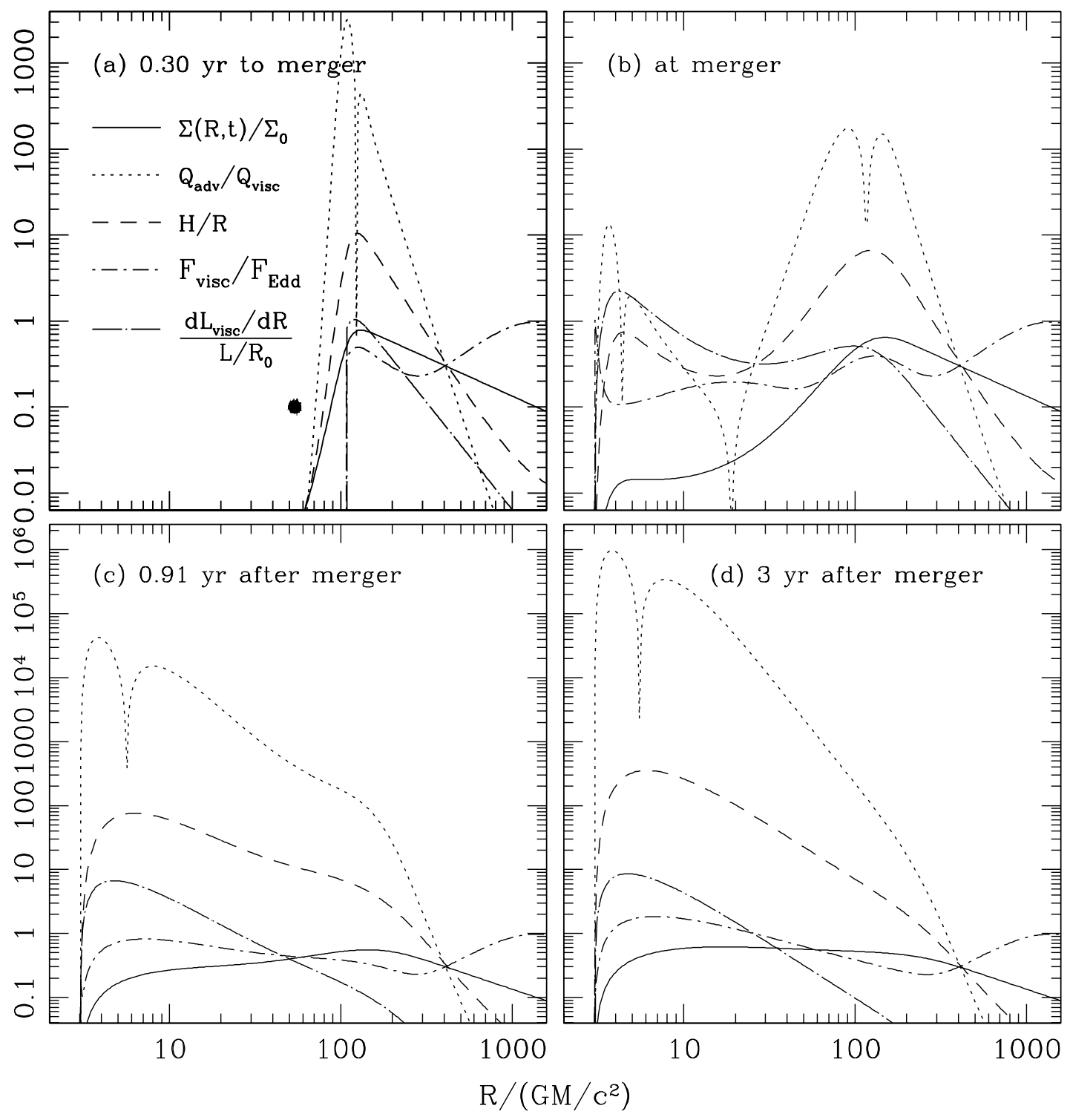

Fig. 7.- Same as Figure 5, but for a more massive and geometrically thicker disk with $S=5$ and $\zeta=1 / 3$. The disk is geometrically thick and advection-dominated in the inner regions of interest, from decoupling to several years after the merger. The local flux also exceeds the Eddington limit at late times (Panel d). 


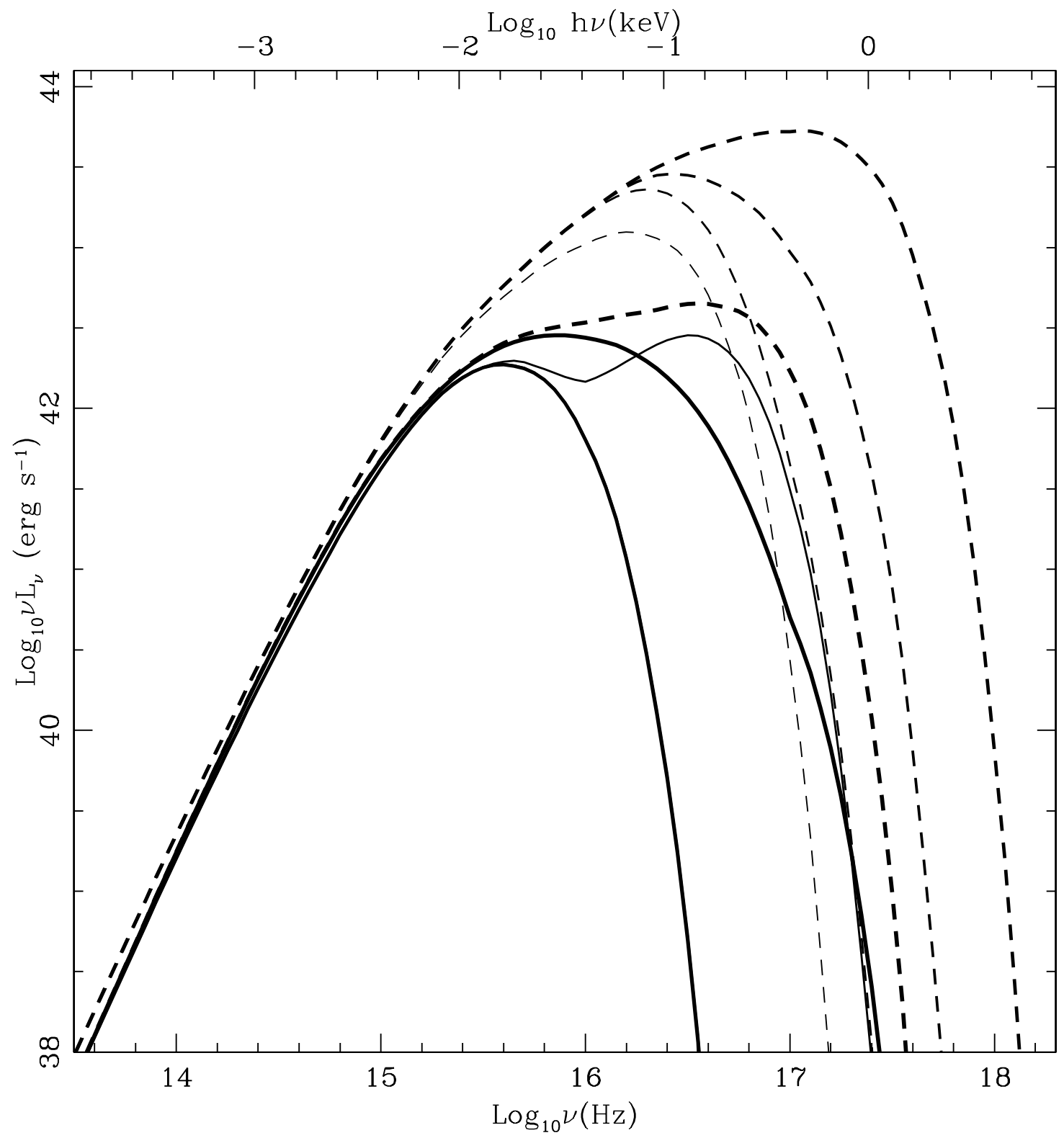

Fig. 8. - The spectra of a more massive and geometrically thick disk, with $S=5$ and $\zeta=1 / 3$, at decoupling and at 1 week, 3 months, $0.91 \mathrm{yr}=t_{\mathrm{EM}}$ and $3 \mathrm{yr}$ after the merger. As in Figure 6, later spectra are shown with thicker lines. The dashed lines show spectra ignoring advective effects, while the solid lines show spectra under the assumption that advection suppresses the emitted flux. In the latter case, the disk is geometrically thick and radiatively inefficient across the entire region inside the initial gap radius, and thus no obvious afterglow may be observable (all curves overlap except the one 1 week after merger, when our calculations show a brief afterglow that is quickly suppressed by advection). 
the midplane temperature at the inner edge, $T_{0,6}$ :

$$
\begin{aligned}
a_{0} & \approx 136 \frac{G M}{c^{2}} \times T_{0,6}^{-2 / 7} \alpha_{-1}^{-2 / 7} \lambda^{1 / 7}\left(\beta_{-1} \zeta\right)^{2 / 7} \\
t_{\mathrm{EM}} & =\frac{3}{4} \beta t_{\mathrm{visc}, 0} \approx 13 \mathrm{yr} \times T_{0,6}^{-8 / 7} \alpha_{-1}^{-8 / 7} \lambda^{4 / 7} M_{6} \beta_{-1}^{8 / 7} \zeta^{1 / 7} \\
\nu_{0} & \approx 2.0 \times 10^{17} \mathrm{~cm}^{2} \mathrm{~s}^{-1} \times T_{0,6}^{4 / 7} \alpha_{-1}^{4 / 7} \lambda^{12 / 7} M_{6}\left(\beta_{-1} \zeta\right)^{3 / 7} \\
\Omega_{0} & \approx 4.5 \times 10^{-5} \mathrm{~s}^{-1} \times T_{0,6}^{3 / 7} \alpha_{-1}^{3 / 7} \lambda^{-12 / 7} M_{6}^{-1}\left(\beta_{-1} \zeta\right)^{-3 / 7}
\end{aligned}
$$

We parametrize the surface density via the arbitrary relation $\Sigma_{0} \equiv S \dot{M}_{\text {Edd }} /\left(3 \pi \nu_{0}\right)$, where the parameter $S$ may be interpreted as the product of the outer mass supply rate in Eddington units and the excess in surface density near the inner edge over the value expected for a standard Shakura-Sunyaev disk, which arises from mass accumulation near the binary (Ivanov et al. 1999; Chang et al. 2009). The latter factor depends on tidal interactions and prior accretion history, and in general is expected to exceed unity. We obtain

$$
\Sigma_{0} \approx 7.3 \times 10^{5} \mathrm{~g} \mathrm{~cm}^{-2} \times T_{0,6}^{-4 / 7} \alpha_{-1}^{-4 / 7} S \lambda^{-12 / 7}\left(\beta_{-1} \zeta\right)^{-3 / 7}
$$

The pressure near the edge is dominated by the radiation pressure $P_{\text {rad }}=(4 \sigma / 3 c) T^{4}$, if $T \gtrsim$ $10^{6} \mathrm{~K}$. We calculate the scale-height-to-radius ratio $H_{0} / R_{0}$ and the radiation-to-gas pressure ratio $P_{\text {rad }, 0} / P_{\text {gas }, 0}$ at the disk edge:

$$
\begin{aligned}
\frac{H_{0}}{R_{0}} & \approx \sqrt{\frac{\gamma P_{\mathrm{rad}, 0} H_{0}}{\Sigma_{0}}} \frac{1}{\Omega_{0} R_{0}}=\frac{\gamma P_{\mathrm{rad}, 0}}{\Sigma_{0} \Omega_{0}^{2} R_{0}} \\
& \approx 0.056 \times T_{0}^{4} S^{-1} \lambda^{4} M_{6} \beta_{-1} \zeta \\
P_{\mathrm{gas}, 0} & =\frac{k T_{0} \Sigma_{0}}{\mu m_{\mathrm{p}} H_{0}}=\frac{k T_{0} \Sigma_{0}^{2} \Omega^{2}}{\gamma \mu m_{\mathrm{p}} P_{\mathrm{rad}, 0}} \\
\frac{P_{\mathrm{rad}, 0}}{P_{\mathrm{gas}, 0}} & =\frac{\gamma \mu m_{\mathrm{p}} P_{\mathrm{rad}, 0}^{2}}{k T_{0} \Sigma_{0}^{2} \Omega^{2}} \\
& \approx 57 \times T_{0,6}^{51 / 7} \alpha_{-1}^{2 / 7} S^{-2} \lambda^{48 / 7} M_{6}^{2}\left(\beta_{-1} \zeta\right)^{12 / 7} .
\end{aligned}
$$

Above, $\gamma \approx 4 / 3$ is the relevant adiabatic index, and $\rho=\Sigma / H$ is the gas density. (Note that in the $H / R$ profiles plotted in Figures [5 and 7, we solve for the scale height $H$ in terms of the total pressure $P_{\text {gas }}+P_{\text {rad }}$ and do not assume radiation pressure dominance.)

\section{A2. Calculating the midplane temperature}

We now turn to calculating the midplane temperature of the inner edge of the disk at decoupling. The thermal spectrum of the inner gas disk differs from that of a blackbody because 
scattering is a significant source of opacity. In such a medium, photons travel a shorter effective path before becoming thermalized and the absorption opacity is effectively enhanced as (e.g., Rybicki \& Lightman 1986; Blaes 2004):

$$
\kappa_{\mathrm{eff}, \nu} \approx \kappa_{\mathrm{abs}, \nu} \sqrt{1+\frac{\kappa_{\mathrm{es}}}{\kappa_{\mathrm{abs}, \nu}}}
$$

where $\kappa_{\mathrm{abs}, \nu}$ and $\kappa_{\mathrm{es}} \approx 0.40 \mathrm{~cm}^{2} \mathrm{~g}^{-1}$ are the absorption and scattering opacities, respectively. The second approximation in equation (A10) is applicable if electron scattering is the dominant source of opacity, which is generally the case for our problem in the spectral range of interest (UV frequencies and higher).

The disk radiates through a photosphere, with photons of different energies being thermalized at different depths. The bottom of the photosphere is defined as the height where the effective optical depth $\tau_{\text {eff }, \nu} \approx \rho_{\mathrm{p}} H_{\mathrm{p}} \kappa_{\text {eff }, \nu}=1$, where $\rho_{\mathrm{p}}$ is the density there and $H_{\mathrm{p}}$ is the scale height of the photosphere. Characterizing $H_{\mathrm{p}}$ as the ratio of the sound speed at the bottom of the photosphere to the orbital angular speed leads to

$$
\rho_{\mathrm{p}} \approx \frac{3 c \Omega^{2}}{4 \gamma \sigma T_{\mathrm{p}}^{4} \kappa_{\mathrm{abs}, \nu}\left(\kappa_{\mathrm{abs}, \nu}+\kappa_{\mathrm{es}}\right)},
$$

where $T_{\mathrm{p}}$ is the temperature at the bottom of the photosphere.

The main source of absorption is the bound-free process, which in general is a complicated function of composition and photon frequency due to its dependence on the ionization state of the gas. For consistency and simplicity, we prescribe the absorption opacity in the same way as MP05, so that the frequency dependence has the same functional form as the free-free absorption opacity, viz.

$$
\kappa_{\mathrm{abs}, \nu} \approx \kappa_{\mathrm{abs}, \mathrm{R}} \rho_{\mathrm{p}} T_{\mathrm{p}}^{-7 / 2}\left[187 f_{\nu}(\xi)\right]
$$

Above, $\xi \equiv h \nu / k T_{\mathrm{p}}, f_{\nu}(\xi) \equiv \xi^{-3}\left(1-e^{-\xi}\right)$ gives the frequency dependence, and $\rho_{\mathrm{p}}$ and $T_{\mathrm{p}}$ are in cgs. The coefficient $\kappa_{\text {abs }, \nu}$ is scaled so that its Rosseland mean yields the Kramer's opacity scaling at solar metallicity, with $\kappa_{\mathrm{abs}, \mathrm{R}} \approx 1.6 \times 10^{24} \mathrm{~cm}^{2} \mathrm{~g}^{-1}$ and the factor in square brackets having a Rosseland mean of unity. Substituting for the photospheric density using equation (A11) gives a cubic equation for the absorption-to-scattering opacity ratio $K_{\nu} \equiv \kappa_{\mathrm{abs}, \nu} / \kappa_{\mathrm{es}}$ at the bottom of the photosphere:

$$
\kappa_{\mathrm{abs}, \nu}^{2}\left(1+\frac{\kappa_{\mathrm{abs}, \nu}}{\kappa_{\mathrm{es}}}\right) \approx 140 \frac{c \kappa_{\mathrm{abs}, \mathrm{R}}}{\gamma \sigma \kappa_{\mathrm{es}}} \Omega^{2} T_{\mathrm{p}}^{-15 / 2} f_{\nu}(\xi) .
$$

We find that in most cases, the vast majority of the disk emission is emitted from in frequencies and regions that are scattering dominated, i.e. $\kappa_{\mathrm{abs}, \nu} / \kappa_{\mathrm{es}} \ll 1$. In this limit, (A13) may be solved trivially to obtain $\kappa_{\mathrm{abs}, \nu} \approx \kappa_{\mathrm{abs}, *} f_{\nu}^{1 / 2}$, where we have defined for convenience the frequency-independent quantity $\kappa_{\mathrm{abs}, *} \approx 4.7 \times 10^{20} \mathrm{~cm}^{2} \mathrm{~g}^{-1} \times(\Omega \mathrm{s})\left(T_{\mathrm{p}} / \mathrm{K}\right)^{-15 / 4}$. However, in some of our solutions there is 
significant emission from regions with $\kappa_{\mathrm{abs}, \nu} / \kappa_{\mathrm{es}} \gtrsim 1$. All of the spectra and radial disk profiles in our figures were computed by solving (A13) generally.

The angle-integrated emergent spectral flux (e.g., Blaes 2004) is

$$
F_{\nu}=\pi \frac{2 \epsilon_{\nu}^{1 / 2}}{1+\epsilon_{\nu}^{1 / 2}} B_{\nu}
$$

where $B_{\nu}$ is the Planck function and $\epsilon_{\nu} \equiv \kappa_{\mathrm{abs}, \nu} /\left(\kappa_{\mathrm{abs}, \nu}+\kappa_{\mathrm{es}}\right)=\left(1+K_{\nu}^{-1}\right)^{-1} \leq 1$ is the frequencydependent ratio of the absorption opacity to the total opacity. Both $\epsilon_{\nu}$ and $B_{\nu}$ are evaluated at the bottom of the photosphere. Each face of the disk contributes half of the total flux $F$. Integrating equation (A14), we obtain:

$$
\frac{F}{2}=\int_{0}^{\infty} F_{\nu} d \nu=\Xi \sigma T_{\mathrm{p}}^{4},
$$

where $\Xi$ is the deviation of the flux from blackbody,

$$
\Xi \equiv \frac{15}{\pi^{4}} \int_{0}^{\infty} \frac{2 \epsilon_{\nu}^{1 / 2}}{1+\epsilon_{\nu}^{1 / 2}(\xi)} \frac{e^{-\xi} d \xi}{f_{\nu}(\xi)}
$$

$\Xi$ may be expressed as a function of the frequency-independent quantity $\epsilon_{*}\left(T_{\mathrm{p}}, \Omega\right) \equiv \kappa_{\mathrm{abs}, *} /\left(\kappa_{\mathrm{abs}, *}+\right.$ $\left.\kappa_{\mathrm{es}}\right) \leq 1$. In the limit $\epsilon_{\nu}^{1 / 2} \ll 1, \Xi \approx 30 \pi^{-4} \epsilon_{*}^{1 / 2} \int_{0}^{\infty} f_{\nu}^{-3 / 4} e^{-\xi} d \xi \approx 0.873 \epsilon_{*}^{1 / 2}$. In the opposite limit $\epsilon_{*} \approx 1$ (i.e. very close to blackbody), $\Xi \approx 1 /\left[1+\left(\epsilon_{*}^{-1}-1\right)^{2 / 3}\right]$. We find that the brightest regions of our disk generally span the entire range $0 \leq \epsilon_{*} \leq 1$. We find a useful fitting formula for $\Xi$ to be

$$
\Xi \approx \frac{0.873 \epsilon_{*}^{-1 / 6}}{1-0.127 \epsilon_{*}^{5 / 6}} \frac{1}{1+\left(\epsilon_{*}^{-1}-1\right)^{2 / 3}} .
$$

The above prescription evaluates $\Xi$ accurately to within $\sim 1 \%$ for the entire range of $\epsilon_{*}$ while reproducing the asymptotic behavior at the extreme limits $\epsilon_{*} \ll 1$ and $\epsilon_{*} \approx 1$. We also find that a much simpler formula, $\Xi \approx(4 / 5) \epsilon_{*}^{1 / 2}$, calculates $\Xi$ accurately to within $10 \%$ for $0 \leq \epsilon \lesssim 0.9$.

The optical depth between the bottom of the photosphere and the midplane is dominated by electron scattering, and thus has negligible frequency dependence. Provided that this region is optically thick and can be described by a one-zone formalism, the midplane and photosphere temperatures are related by $T_{\mathrm{p}}^{4}=(4 / 3) T^{4} / \tau$, where $\tau$ is the optical thickness between the two heights. Following MP05, we write $\tau=\theta \kappa_{\mathrm{es}} \Sigma$, where $\theta=\theta_{0.2} / 0.2 \leq 1$ is a porosity correction factor. Ignoring horizontal advection, we equate $F$ with the power viscously dissipated per unit area in the disk:

$$
F=2 \times \Xi\left(\epsilon_{*}\right) \frac{4 \sigma T^{4}}{3 \tau} \approx \frac{32 \sigma T^{4} \epsilon_{*}^{1 / 2}}{15 \tau} \sim \frac{9}{4} \nu \Sigma \Omega^{2} .
$$


We now solve for $T_{0,6}$ by substituting equations (A5), (A6) and (A7) into equation (A18). A useful intermediate result is

$$
\epsilon_{*}^{1 / 2} \approx \sqrt{\frac{\kappa_{\mathrm{abs}, *}(\Omega, T)}{\kappa_{\mathrm{es}}}} \approx 0.19 T_{0,6}^{-27 / 14} \times \alpha_{-1}^{-3 / 56} S^{15 / 32} \lambda^{-93 / 56} M_{6}^{-1 / 2}\left(\beta_{-1} \zeta\right)^{-93 / 224} \theta_{0.2}^{15 / 32}
$$

which gives $T_{0,6} \propto\left(\nu_{0} \Sigma_{0} \Omega_{0}^{2} \epsilon_{*}^{-1 / 2} \tau_{0}\right)^{14 / 25}$. That is, the midplane temperature scales with the flux scaling to the $+14 / 25$ th power, i.e. more sensitively than in the standard black body relation of $T \propto F_{\mathrm{bb}}^{1 / 4}$. We finally arrive at

$$
T_{0,6} \approx 1.3 \times \alpha_{-1}^{19 / 100} S^{343 / 400} \lambda^{-39 / 20} M_{6}^{-7 / 25}\left(\beta_{-1} \zeta\right)^{-39 / 80} \theta_{0.2}^{119 / 400}
$$

We derive an inner-edge temperature that is somewhat lower than the value calculated by MP05, primarily because their prescription for the emitted flux is lower than ours by a factor of two. (Compare their expression for $F_{\nu}$ with our equation (A14). See also our discussion that follows equation (3.4).) As noted above, the midplane temperature is rather sensitive to the flux. We calculate that MP05 underestimated the flux through the omission of the factor of two, and slightly overestimated the frequency integral. Overall, we calculate a bolometric flux $60 \%$ higher than they did, and our disk temperature is lower by a factor $1.6^{14 / 25} \approx 1.3$. We derive identical power-law dependencies on the system parameters as they did.

The lower temperature suggests a slightly longer timescale for the nominal onset of the X-ray afterglow after merger, $t_{\mathrm{EM}} \sim(3 / 4) \beta t_{\mathrm{visc}, 0} \propto T_{0}^{-8 / 7} \sim 9 \times M_{6}^{1.3} \mathrm{yr}$ for fiducial parameters. We also calculate lower values for the quantities $H_{0} / R_{0}$ and $P_{\text {rad }, 0} / P_{\text {gas }, 0}$, which are highly sensitive to the disk temperature (equations [A8] and [A9]) and thus significantly reduced when the factor of two correction to the flux is included. We summarize our results below, and in our Table 1.

$$
\begin{aligned}
a_{0} & \approx 126 \frac{G M}{c^{2}} \times \alpha_{-1}^{-17 / 50} S^{-49 / 200} \lambda^{7 / 10} M_{6}^{2 / 25}\left(\beta_{-1} \zeta\right)^{17 / 40} \theta_{0.2}^{-17 / 200} \\
\Omega_{0} & \approx 5.1 \times 10^{-5} \mathrm{~s}^{-1} \times \alpha_{-1}^{51 / 100} S^{147 / 400} \lambda^{-51 / 20} M_{6}^{-28 / 25}\left(\beta_{-1} \zeta\right)^{-51 / 80} \theta_{0.2}^{51 / 400} \\
t_{\mathrm{EM}} & \approx 9.2 \mathrm{yr} \times \alpha_{-1}^{-34 / 25} S^{-49 / 50} \lambda^{14 / 5} M_{6}^{33 / 25} \beta_{-1}^{17 / 10} \zeta^{7 / 10} \theta_{0.2}^{-17 / 50} \\
\Sigma_{0} & \approx 6.2 \times 10^{5} \mathrm{~g} \mathrm{~cm}{ }^{-2} \times \alpha_{-1}^{-17 / 25} S^{51 / 100} \lambda^{-3 / 5} M_{6}^{4 / 25}\left(\beta_{-1} \zeta\right)^{-3 / 20} \theta_{0.2}^{-17 / 100} \\
\frac{H_{0}}{R_{0}} & \approx 0.17 \times \alpha_{-1}^{19 / 25} S^{243 / 100} \lambda^{-19 / 5} M_{6}^{-3 / 25}\left(\beta_{-1} \zeta\right)^{-19 / 20} \theta_{0.2}^{119 / 100} \\
\frac{P_{\mathrm{rad}, 0}}{P_{\text {gas }, 0}} & \approx 430 \times \alpha_{-1}^{167 / 100} S^{1699 / 400} \lambda^{-147 / 20} M_{6}^{-1 / 25}\left(\beta_{-1} \zeta\right)^{-147 / 80} \theta_{0.2}^{867 / 400} \\
\epsilon_{*}^{1 / 2} & \approx 0.11 \times \alpha_{-1}^{-21 / 50} S^{-237 / 200} \lambda^{21 / 10} M_{6}^{1 / 25}\left(\beta_{-1} \zeta\right)^{21 / 40} \theta_{0.2}^{-21 / 200}
\end{aligned}
$$

We calculate a somewhat shorter scale height for the disk than MP05, who had derived $H_{0} / R_{0} \sim$ 0.46 for fiducial parameters. We show in $\$ 3.4$ that the advected flux at decoupling is not likely to be significant in the circumbinary disk (for $S=1$ ). 


\section{A3. Prescribing a value for the viscosity power-law index $n$}

In our model we prescribe a simple radial power-law $\nu \propto R^{n}$ for the viscosity. In the $\alpha_{\text {gas }}$-disk model, however, $\nu$ has a physical definition with $\nu \propto \alpha P_{\text {gas }} /(\rho \Omega) \propto T / \Omega$. This suggests that a reasonable value for $n$ should satisfy the relation

$$
n \approx \frac{d \ln T}{d \ln r}+3 / 2
$$

Below, we apply the above relationship between $\nu$ and $T$ to evaluate an appropriate value for our viscosity power-law index $n$.

For fiducial parameters, near the disk edge at decoupling and inside this radius thereafter, $\Xi \approx(4 / 5) \epsilon_{*}^{1 / 2}$ and $\epsilon_{*} \approx \kappa_{\mathrm{abs}, *} / \kappa_{\mathrm{es}} \propto \Omega T_{\mathrm{p}}^{-15 / 4}$. Applying these approximations to equation (A18) leads to $T_{\mathrm{p}}^{17 / 8} \propto \nu \Sigma \Omega^{3 / 2}$. Since $T \propto \tau^{1 / 4} T_{\mathrm{p}} \propto \Sigma^{1 / 4} T_{\mathrm{p}}$, we obtain

$$
T(r, t) \propto\left[\frac{\Sigma(r, t)}{\Sigma_{0}}\right]^{49 / 68} r^{(8 n-18) / 17}
$$

One way of characterizing the accretion flow is through the power-law index $m \equiv d \ln (\nu \Sigma) / d \ln R$. Writing $d \ln \Sigma / d \ln R=m-n$ and substituting this into equations (A28) and (A29) leads to the solution $n \approx(30+49 m) / 85$. If the disk just outside the inner edge behaves like a steady-state accretion solution with $m \approx 0$, then equation (A28) suggests that in this region a reasonable value of $n$ is $6 / 17 \approx 0.35$. If, however, the gas in this region behaves more like a decretion profile with $m \approx-1 / 2$, then $n=11 / 170 \approx 0.065$ would be more appropriate.

The disk surface density profile outside the edge at decoupling, however, is not particularly relevant to the viscous refilling rate of the central cavity. Rather, the pertinent value of $n$ is that where the torque gradient is highest, where the most rapid evolution occurs, i.e. near the inner edge of the disk. However, in this region $d \ln (T / \Omega) / d \ln R>0$ is a rapidly changing function of time and radius. Thus, there is no single value for $n$ that can fully describe the viscous evolution of an $\alpha$-disk. At late times ( $t \gtrsim t_{\text {visc }}(R)$ after the merger), however, the inner disk evolves toward a standard quasisteady accretion solution with $\nu \Sigma \propto\left(1-\sqrt{R_{*} / R}\right)$, where $R_{*}$ is the inner boundary radius imposed by the MBH remnant. In this limit, equation (A28) leads to $n \approx 6 / 17+49 /\left(170 / \sqrt{R / R_{*}-1}\right)$, which evaluates to $n \approx 0.4$ at radii $R \lesssim R_{0}$ for $1 \leq R_{*} c^{2} / G M \leq 6$. We thus select $n=0.4$ as our fiducial viscosity power-law index, as it is consistent with the asymptotic evolution of the inner disk at late times.

Upon experimenting with several values for $n$, we have found that the choice of $n$ does not qualitatively affect the main conclusions of our study, as long as $n \lesssim 1$. The viscous evolution is driven by the single timescale $\tau \propto t_{\text {visc }} /(1-n / 2)^{2}$, and the bolometric and monochromatic luminosities also have weak direct dependencies on $n$. 


\section{B. Green's Function for the Viscous Evolution of the Disk Surface Density}

Equation (2.3), a second-order diffusion equation, is linear if $\nu$ does not depend on $\Sigma$. Below, we follow the formalism of Lynden-Bell \& Pringle (1974; see also Ogilvie 2005) to derive a Green's function solution for the viscous evolution.

Suppose the solution has the exponentially decaying form $\Sigma(R, t)=\exp (-\Lambda t) R^{p} \varsigma(R)$, where $\varsigma(R)$ is an arbitrary function of radius and $p$ is an arbitrary real number. With the additional assumption that $\nu$ behaves as a power-law, $\nu=\nu_{0}\left(R / R_{0}\right)^{n}$, equation (2.3) may be rewritten as a modified Bessel equation:

$$
R^{2} \frac{\partial^{2} \varsigma}{\partial R^{2}}+\left(2 p+2 n+\frac{3}{2}\right) R \frac{\partial \varsigma}{\partial R}+\left[(p+n)\left(\frac{\Lambda}{3 \nu_{0}} R^{2-n}+p+n+\frac{1}{2}\right)\right] \varsigma=0 .
$$

Selecting $p=n-1 / 4$ for convenience and making the substitution $\Lambda=3 \nu_{0} k^{2} R_{0}^{-n}$, the equation above has a set of solutions

$$
\varsigma_{k}(R)=R^{-2 n}\left[A(k) J_{1 /(4-2 n)}\left(\frac{k R^{1-n / 2}}{1-n / 2}\right)+B(k) Y_{1 /(4-2 n)}\left(\frac{k R^{1-n / 2}}{1-n / 2}\right)\right],
$$

where $n<2, J$ and $Y$ are the ordinary Bessel functions of the first and second kind, respectively, $k>0$ is their mode, and $A(k)$ and $B(k)$ are arbitrary coefficients for each mode.

We note here that even with a simple prescription for $\nu$, the general problem of the viscous evolution of a circumbinary disk around a binary is not easily treated analytically. For a case where the Green's function is particularly simple, Pringle (1991) showed that one may account for the binary torques by applying a zero-torque boundary condition $\partial\left(\nu \Sigma R^{1 / 2}\right) / \partial R=0$ at the gap opening radius. For binaries of interest in this paper, the gap-opening radius itself is a function of time, which greatly adds to the "extreme algebraic complexity" (Pringle 1991) that is generally involved with applying such a boundary condition at nonzero radius. Our problem, however, is greatly simplified by the decoupling condition, which allows us to approximate the early evolution as if the circumbinary disk experiences no torques from the binary. We thus proceed as if the potential is due to a single central point mass, and approximate the gas orbits as being circular and Keplerian. We discuss the justifications and the modest evolutionary effects associated with this model simplification in $\$ 2.2$.

The boundary condition of interest, then, is that the torques vanish at the center. (At late times, it will be necessary to consider the inner boundary condition imposed at finite radius by the central $\mathrm{MBH}$ remnant. We shall address this point shortly.) Because $Y_{1 /(4-2 n)}(k y)$ diverges as $y \rightarrow 0$, our physical solution requires all $B(k)$ to vanish. The general solution is a sum of all possible modes over all $k \geq 0$ weighted by a set of coefficients $A(k)$, viz.:

$$
\Sigma(R, t)=R^{-n-1 / 4} \int_{0}^{\infty} A(k) J_{1 /(4-2 n)}(k y) \exp \left[-3 \nu_{0} R_{0}^{-n} k^{2} t\right] d k
$$


where we have defined $y \equiv R^{1-n / 2} /(1-n / 2)$ for convenience.

The function $A(k)$ can be evaluated through the use of Hankel transforms. For a given order of the Bessel function $\ell$, Hankel transform pairs satisfy:

$$
\begin{aligned}
\Phi(R) & =\int_{0}^{\infty} \phi(k) J_{\ell}(k R) k d k \\
\phi(k) & =\int_{0}^{\infty} \Phi(R) J_{\ell}(k R) R d R
\end{aligned}
$$

For our problem, we may construct the Hankel transform pair

$$
\begin{aligned}
& \Phi(y)=R^{n+1 / 4} \Sigma(y, 0)=\int_{0}^{\infty}\left[k^{-1} A(k)\right] J_{1 /(4-2 n)}(k y) k d k \\
& \phi(k)=k^{-1} A(k)=\int_{0}^{\infty}\left[R^{\prime n+1 / 4} \Sigma\left(y^{\prime}, 0\right)\right] J_{1 /(4-2 n)}\left(k y^{\prime}\right) y^{\prime} d y^{\prime}
\end{aligned}
$$

from which we obtain

$$
\begin{aligned}
A(k) & =\int_{0}^{\infty} R^{\prime n+1 / 4} \Sigma\left(y^{\prime}, 0\right) J_{1 /(4-2 n)}\left(k y^{\prime}\right) k y^{\prime} d y^{\prime} \\
& =\left(1-\frac{n}{2}\right)^{-1} \int_{0}^{\infty} \Sigma\left(y^{\prime}, 0\right) J_{1 /(4-2 n)}\left(k y^{\prime}\right) k R^{\prime 5 / 4+n / 2} d R^{\prime}
\end{aligned}
$$

Combining equations ( $\mathrm{B} 3)$ and $(\mathrm{B} 6)$, we may write the solution $\Sigma(y, t)$ as an integral function of the initial condition $\Sigma\left(R^{\prime}, 0\right)$,

$$
\begin{aligned}
\Sigma(R, t)= & \left(1-\frac{n}{2}\right)^{-1} R^{-n-1 / 4} \int_{0}^{\infty} R^{\prime 5 / 4+n / 2} \int_{0}^{\infty} \Sigma\left(R^{\prime}, t=0\right) \exp \left(-3 \nu_{0} R_{0}^{-n} k^{2} t\right) \\
& \quad \times J_{1 /(4-2 n)}\left(k y^{\prime}\right) J_{1 /(4-2 n)}(k y) k d k d R^{\prime} \\
\equiv & \int_{0}^{\infty} G_{0}\left(R, R^{\prime}, t\right) \Sigma\left(R^{\prime}, t=0\right) \frac{d R^{\prime}}{R_{0}},
\end{aligned}
$$

with the Green's function

$$
\begin{aligned}
G_{0} & =\left(1-\frac{n}{2}\right)^{-1} R^{-1 / 4-n} R^{15 / 4} R_{0} \int_{0}^{\infty} J_{1 /(4-2 n)}\left(k y^{\prime}\right) J_{1 /(4-2 n)}(k y) \exp \left(-3 \nu_{0} R_{0}^{-n} k^{2} t\right) k d k \\
& =\frac{R^{-1 / 4-n} R^{\prime 5 / 4} R_{0}^{1+n}}{6(1-n / 2) \nu_{0} t} I_{1 /(4-2 n)}\left[\frac{R^{1-n / 2} R^{\prime 1-n / 2} R_{0}^{n}}{6(1-n / 2)^{2} \nu_{0} t}\right] \exp \left[-\frac{\left(R^{2-n}+R^{\prime 2-n}+\right) R_{0}^{n}}{12(1-n / 2)^{2} \nu_{0} t}\right] \\
& =\frac{2-n}{\tau} r^{-1 / 4-n} r^{\prime 5 / 4} I_{1 /(4-2 n)}\left(\frac{2 r^{1-n / 2} r^{\prime 1-n / 2}}{\tau}\right) \exp \left(-\frac{r^{2-n}+r^{\prime 2-n}}{\tau}\right),
\end{aligned}
$$

where $I_{\ell}$ is the modified Bessel function of the first kind and order $\ell$, and we have substituted the dimensionless variables $r \equiv R / R_{0}$ and $\tau \equiv 8(1-n / 2)^{2} t / t_{\text {visc }, 0}$. 
In deriving the Green's function above, we have applied the zero-torque boundary condition at the origin, instead of at some finite boundary radius $R_{*}$. At late times and small radii, $\tau \gg r^{2-n}$, the solutions obtained using equation ( $(\mathrm{B} 8)$ have the radial dependence $\partial \ln (\nu \Sigma) / \partial \ln R \approx(n-2) r^{2-n} / \tau$, viz. they converge to a quasi-steady profile with $\nu \Sigma \propto \Sigma r^{n}$ constant in radius. Thus, $\Sigma$ diverges at the center at late times if $n$ is positive.

A physically plausible model should account for the boundary condition imposed by the $\mathrm{MBH}$ remnant at late times. Below, we approximate the Green's function for the case where the zerotorque boundary condition is applied at a finite boundary radius $R_{*}>0$. In this case the boundary condition determines the relationship between the $A(k)$ and $B(k)$ coefficients in equation ( $(\bar{B} 2)$, viz:

$$
\varsigma_{k}(R)=R^{-2 n} A(k)\left[J_{1 /(4-2 n)}(k y)-\frac{J_{1 /(4-2 n)}\left(k y_{*}\right)}{Y_{1 /(4-2 n)}\left(k y_{*}\right)} Y_{1 /(4-2 n)}(k y)\right],
$$

where $y_{*}=R_{*}^{1-n / 2} /(1-n / 2)$.

Our new Green's function is then

$$
\begin{aligned}
G=R^{-1 / 4-n} & R^{5 / 4+n / 2} \int_{0}^{\infty} J_{1 /(4-2 n)}(k y) J_{1 /(4-2 n)}\left(k y^{\prime}\right) \exp \left[-\frac{R_{0}^{2-n} k^{2}}{4(1-n / 2)^{2}} \tau\right] \\
\times & {\left[1-\frac{J_{1 /(4-2 n)}\left(k y_{*}\right)}{Y_{1 /(4-2 n)}\left(k y_{*}\right)} \frac{Y_{1 /(4-2 n)}(k y)}{J_{1 /(4-2 n)}(k y)}\right] k d k . }
\end{aligned}
$$

The integrand above is greatest where $k$ is small, and decays rapidly as $k$ increases beyond $k^{2} \sim$ $4 R_{0}^{n-2} \tau^{-1}$. The boundary condition, however, has a significant effect on the viscous evolution only where $\tau \gg r^{2-n}=y^{2} R_{0}^{n-2}$ and $y \gtrsim y_{*}$. Thus, the boundary effect is appreciable only where $k^{2} \ll$ $4 y_{*}^{-2} \gtrsim 4 y^{-2}$. In the limit of small argument $J_{\ell}(x) / Y_{\ell}(x) \propto x^{2 m}$, and we obtain

$$
\frac{J_{1 /(4-2 n)}\left(k y_{*}\right)}{Y_{1 /(4-2 n)}\left(k y_{*}\right)} \frac{Y_{1 /(4-2 n)}(k y)}{J_{1 /(4-2 n)}(k y)} \approx\left(\frac{y_{*}}{y}\right)^{1 /(2-n)}=\sqrt{\frac{R_{*}}{R}} .
$$

To leading order, then, at late times and small radii the factor in square brackets in equation (B10) evaluates to $1-\sqrt{R_{*} / R}$. This factor may be taken outside of the integral, and gives the asymptotic behavior $\nu \Sigma \propto 1-\sqrt{R_{*} / R}$, which is precisely the standard solution for a steady thin accretion disk with a no-torque inner boundary at $R_{*}$ (e.g., Frank et al. 2002).

We do not evaluate the exact Green's function solution at early times or at large radii, where the effect of the boundary condition is minimal. Instead, we approximate the function by ensuring the correct boundary behavior at late times and small radii, as suggested by Lynden-Bell \& Pringle (1974). Accounting for the fact that the boundary at $R_{*}$ exists only after the time of merger, which evaluates via the decoupling condition to $\tau_{\text {merge }}=2 \beta(1-n / 2)^{2}$, we approximate our new Green's 
function as follows:

$$
G\left(r, r^{\prime}, \tau\right)=\left\{1-\sqrt{\frac{r_{*}}{r}} \exp \left[-\frac{\left(r-r_{*}\right)^{2-n}}{\mathcal{R}\left(\tau-\tau_{\text {merge }}\right)}\right]\right\} G_{0}\left(r, r^{\prime}, \tau\right),
$$

where $G_{0}$ is the Green's function given in equation ( $\mathrm{B} 8$ ) for the case $R_{*}=0, r_{*} \equiv R_{*} / R_{0}$, and $\mathcal{R}$ is the ramp function, defined as $\mathcal{R}(x \leq 0)=0$ and $\mathcal{R}(x>0)=x$.

\section{REFERENCES}

Abramowicz, M. A., Czerny, B., Lasota, J. P., \& Szuszkiewicz, E. 1988, ApJ, 332, 646

Anderson, M., Lehner, L., Megevand, M., \& Neilsen, D. 2009, ArXiv e-prints, astro-ph/0910.4969

Armitage, P. J. \& Natarajan, P. 2002, ApJ, 567, L9

-. 2005, ApJ, 634, 921

Artemova, I. V., Bjoernsson, G., \& Novikov, I. D. 1996, ApJ, 461, 565

Artymowicz, P., Clarke, C. J., Lubow, S. H., \& Pringle, J. E. 1991, ApJ, 370, L35

Baker, J., Campanelli, M., Lousto, C. O., \& Takahashi, R. 2004, Phys. Rev. D, 69, 027505

Bardeen, J. M. 1970, Nature, 226, 64

Begelman, M. C. 2002, ApJ, 568, L97

Begelman, M. C., Blandford, R. D., \& Rees, M. J. 1980, Nature, 287, 307

Bekenstein, J. D. 1973, ApJ, 183, 657

Berti, E. \& Volonteri, M. 2008, ApJ, 684, 822

Blaes, O. M. 2004, in Accretion Discs, Jets and High Energy Phenomena in Astrophysics, ed. V. Beskin, G. Henri, F. Menard, \& et al., 137-185

Blandford, R. D. \& Begelman, M. C. 1999, MNRAS, 303, L1

Bloom, J. S., Holz, D. E., Hughes, S. A., \& Menou, K. 2009, in The Astronomy and Astrophysics Decadal Survey, Vol. 2010, 20

Bode, N. \& Phinney, S. 2007, APS Meeting Abstracts, 1010 
Callegari, S., Mayer, L., Kazantzidis, S., Colpi, M., Governato, F., Quinn, T., \& Wadsley, J. 2009, ApJ, 696, L89

Chang, P., Strubbe, L. E., Menou, K., \& Quataert, E. 2009, ArXiv e-prints, astro-ph/0906.0825

Colpi, M., Callegari, S., Dotti, M., \& Mayer, L. 2009, Classical and Quantum Gravity, 26, 094029

Corrales, L. R., Haiman, Z., \& MacFadyen, A. 2009, ArXiv e-prints, astro-ph/0910.0014

Cuadra, J., Armitage, P. J., Alexander, R. D., \& Begelman, M. C. 2009, MNRAS, 393, 1423

Cutler, C. 1998, Phys. Rev. D, 57, 7089

Dotti, M., Colpi, M., Haardt, F., \& Mayer, L. 2007, MNRAS, 379, 956

Escala, A., Larson, R. B., Coppi, P. S., \& Mardones, D. 2005, ApJ, 630, 152

Ferrarese, L. 2002, ApJ, 578, 90

Frank, J., King, A., \& Raine, D. J. 2002, Accretion Power in Astrophysics: Third Edition (Cambridge University Press)

Günther, R., Schäfer, C., \& Kley, W. 2004, A\&A, 423, 559

Haehnelt, M. G. 1994, MNRAS, 269, 199

Haiman, Z., Kocsis, B., \& Menou, K. 2009a, ApJ, 700, 1952

-. 2009b, ApJ, 700, 1952

Hayasaki, K., Mineshige, S., \& Ho, L. C. 2008, ApJ, 682, 1134

Hayasaki, K., Mineshige, S., \& Sudou, H. 2007, PASJ, 59, 427

Hirose, S., Blaes, O., \& Krolik, J. H. 2009a, ArXiv e-prints, astro-ph/0908.1117

Hirose, S., Krolik, J. H., \& Blaes, O. 2009b, ApJ, 691, 16

Holz, D. E. \& Hughes, S. A. 2005, ApJ, 629, 15

Hughes, S. A. 2002, MNRAS, 331, 805

Hughes, S. A. \& Blandford, R. D. 2003, ApJ, 585, L101

Ivanov, P. B., Papaloizou, J. C. B., \& Polnarev, A. G. 1999, MNRAS, 307, 79

King, A. R. \& Pringle, J. E. 2006, MNRAS, 373, L90 
Kocsis, B., Frei, Z., Haiman, Z., \& Menou, K. 2006, ApJ, 637, 27

Kocsis, B., Haiman, Z., Menou, K., \& Frei, Z. 2007, Phys. Rev. D, 76, 022003

Kocsis, B. \& Loeb, A. 2008, Physical Review Letters, 101, 041101

Kormendy, J. \& Richstone, D. 1995, ARA\&A, 33, 581

Krolik, J. H. 2010, ApJ, 709, 774

Krolik, J. H. \& Hawley, J. F. 2002, ApJ, 573, 754

Lang, R. N. \& Hughes, S. A. 2006, Phys. Rev. D, 74, 122001

-. 2008, ApJ, 677, 1184

Lightman, A. P. \& Eardley, D. M. 1974, ApJ, 187, L1+

Lippai, Z., Frei, Z., \& Haiman, Z. 2008, ApJ, 676, L5

Liu, F. K. 2004, MNRAS, 347, 1357

Liu, F. K., Wu, X., \& Cao, S. L. 2003, MNRAS, 340, 411

Lodato, G., Nayakshin, S., King, A. R., \& Pringle, J. E. 2009, MNRAS, 398, 1392

Lubow, S. H., Seibert, M., \& Artymowicz, P. 1999, ApJ, 526, 1001

Lynden-Bell, D. \& Pringle, J. E. 1974, MNRAS, 168, 603

MacFadyen, A. I. \& Milosavljević, M. 2008, ApJ, 672, 83

Magorrian, J., Tremaine, S., Richstone, D., Bender, R., Bower, G., Dressler, A., Faber, S. M., Gebhardt, K., Green, R., Grillmair, C., Kormendy, J., \& Lauer, T. 1998, AJ, 115, 2285

Megevand, M., Anderson, M., Frank, J., Hirschmann, E. W., Lehner, L., Liebling, S. L., Motl, P. M., \& Neilsen, D. 2009, Phys. Rev. D, 80, 024012

Menou, K., Haiman, Z., \& Narayanan, V. K. 2001, ApJ, 558, 535

Milosavljević, M. \& Phinney, E. S. 2005, ApJ, 622, L93

Mösta, P., Palenzuela, C., Rezzolla, L., Lehner, L., Yoshida, S., \& Pollney, D. 2009, ArXiv e-prints

Narayan, R. \& Yi, I. 1994, ApJ, 428, L13

-. 1995, ApJ, 452, 710 
Novikov, I. D. \& Thorne, K. S. 1973, in Black Holes (Les Astres Occlus), 343-450

Ogilvie, G. I. 2005, Unpublished lecture notes on "Accretion Discs", http://www.damtp.cam.ac.uk/user/giolo/lecture5.pdf

O’Neill, S. M., Miller, M. C., Bogdanović, T., Reynolds, C. S., \& Schnittman, J. D. 2009, ApJ, 700,859

Palenzuela, C., Anderson, M., Lehner, L., Liebling, S. L., \& Neilsen, D. 2009a, Physical Review Letters, 103, 081101

Palenzuela, C., Lehner, L., \& Yoshida, S. 2009b, ArXiv e-prints, astro-ph/0911.3889

Peres, A. 1962, Physical Review, 128, 2471

Phinney, E. S. 2009, in The Astronomy and Astrophysics Decadal Survey, Vol. 2010, 235

Piran, T. 1978, ApJ, 221, 652

Pringle, J. E. 1976, MNRAS, 177, 65

-. 1981, ARA\&A, 19, 137

-. 1991, MNRAS, 248, 754

Rees, M. J., Begelman, M. C., Blandford, R. D., \& Phinney, E. S. 1982, Nature, 295, 17

Reisswig, C., Husa, S., Rezzolla, L., Dorband, E. N., Pollney, D., \& Seiler, J. 2009, Phys. Rev. D, 80,124026

Richstone, D., Ajhar, E. A., Bender, R., Bower, G., Dressler, A., Faber, S. M., Filippenko, A. V., Gebhardt, K., Green, R., Ho, L. C., Kormendy, J., Lauer, T. R., Magorrian, J., \& Tremaine, S. 1998, Nature, 395, A14+

Rossi, E. M., Lodato, G., Armitage, P. J., Pringle, J. E., \& King, A. R. 2009, ArXiv e-prints, astro-ph/0910.0002

Rudak, B. \& Paczynski, B. 1981, Acta Astronomica, 31, 13

Rybicki, G. B. \& Lightman, A. P. 1986, Radiative Processes in Astrophysics (Wiley-VCH)

Schnittman, J. D. \& Krolik, J. H. 2008, ApJ, 684, 835

Schutz, B. F. 2009, Classical and Quantum Gravity, 26, 094020 
Sesana, A., Volonteri, M., \& Haardt, F. 2007, MNRAS, 377, 1711

Shakura, N. I. \& Sunyaev, R. A. 1973, A\&A, 24, 337

—. 1976, MNRAS, 175, 613

Shapiro, S. L. 2010, Phys. Rev. D, 81, 024019

Shapiro, S. L., Lightman, A. P., \& Eardley, D. M. 1976, ApJ, 204, 187

Shields, G. A. \& Bonning, E. W. 2008, ApJ, 682, 758

Tanaka, T. \& Haiman, Z. 2009, ApJ, 696, 1798

Tichy, W. \& Marronetti, P. 2008, Phys. Rev. D, 78, 081501

Turner, N. J. 2004, ApJ, 605, L45

Vecchio, A. 2004, Phys. Rev. D, 70, 042001

Volonteri, M., Haardt, F., \& Madau, P. 2003, ApJ, 582, 559

Wyithe, J. S. B. \& Loeb, A. 2003, ApJ, 590, 691 